\title{
Hybrid Gradient-Projection Algorithm for Solving Constrained Convex Minimization Problems with Generalized Mixed Equilibrium Problems
}

\author{
Lu-Chuan Ceng' and Ching-Feng Wen ${ }^{2}$ \\ ${ }^{1}$ Department of Mathematics, Scientific Computing Key Laboratory of Shanghai Universities, \\ Shanghai Normal University, Shanghai 200234, China \\ ${ }^{2}$ Center for General Education, Kaohsiung Medical University, Kaohsiung 80708, Taiwan \\ Correspondence should be addressed to Ching-Feng Wen, cfwen@kmu.edu.tw
}

Received 16 July 2012; Accepted 8 September 2012

Academic Editor: Hong-Kun Xu

Copyright (C 2012 L.-C. Ceng and C.-F. Wen. This is an open access article distributed under the Creative Commons Attribution License, which permits unrestricted use, distribution, and reproduction in any medium, provided the original work is properly cited.

\begin{abstract}
It is well known that the gradient-projection algorithm (GPA) for solving constrained convex minimization problems has been proven to have only weak convergence unless the underlying Hilbert space is finite dimensional. In this paper, we introduce a new hybrid gradient-projection algorithm for solving constrained convex minimization problems with generalized mixed equilibrium problems in a real Hilbert space. It is proven that three sequences generated by this algorithm converge strongly to the unique solution of some variational inequality, which is also a common element of the set of solutions of a constrained convex minimization problem, the set of solutions of a generalized mixed equilibrium problem, and the set of fixed points of a strict pseudocontraction in a real Hilbert space.
\end{abstract}

\section{Introduction}

Let $H$ be a real Hilbert space with inner product $\langle\cdot, \cdot\rangle$ and norm $\|\cdot\|$. Let $C$ be a nonempty closed convex subset of $H$ and let $P_{C}$ be the metric projection of $H$ onto $C$. Recall that a $\rho$-Lipschitz continuous mapping $T: C \rightarrow H$ is a mapping on $C$ such that

$$
\|T x-T y\| \leq \rho\|x-y\|, \quad \forall x, y \in C,
$$

where $\rho \geq 0$ is a constant. In particular, if $\rho \in[0,1)$ then $T$ is called a contraction on $C$; if $\rho=1$ then $T$ is called a nonexpansive mapping on $C$. A mapping $A: C \rightarrow H$ is called monotone if

$$
\langle A x-A y, x-y\rangle \geq 0, \quad \forall x, y \in C .
$$


A mapping $A: C \rightarrow H$ is called $\alpha$-inverse strongly monotone if there exists a constant $\alpha>0$ such that

$$
\langle A x-A y, x-y\rangle \geq \alpha\|A x-A y\|^{2}, \quad \forall x, y \in C ;
$$

see, for example, [1]. A self-mapping $S: C \rightarrow C$ is called $k$-strictly pseudocontractive if there exists a constant $k \in[0,1)$ such that

$$
\|S x-S y\|^{2} \leq\|x-y\|^{2}+k\|(I-S) x-(I-S) y\|^{2}, \quad \forall x, y \in C
$$

see, for example, [2]. In particular, if $k=0$, then $S$ reduces to a nonexpansive self-mapping on $C$.

Consider the following constrained convex minimization problem:

$$
\operatorname{minimize}\{f(x): x \in C\} \text {, }
$$

where $f: C \rightarrow \mathbf{R}$ is a real-valued convex function. If $f$ is (Frechet) differentiable, then the gradient-projection method (for short, GPM) generates a sequence $\left\{x_{n}\right\}$ via the recursive formula

$$
x_{n+1}=P_{C}\left(x_{n}-\lambda \nabla f\left(x_{n}\right)\right), \quad \forall n \geq 0,
$$

or more generally,

$$
x_{n+1}=P_{C}\left(x_{n}-\lambda_{n} \nabla f\left(x_{n}\right)\right), \quad \forall n \geq 0,
$$

where in both (1.6) and (1.7), the initial guess $x_{0}$ is taken from $C$ arbitrarily, the parameters, $\lambda$ or $\lambda_{n}$, are positive real numbers, and $P_{C}$ is the metric projection from $H$ onto $C$. The convergence of the algorithms (1.6) and (1.7) depends on the behavior of the gradient $\nabla f$. As a matter of fact, it is known that if $\nabla f$ is strongly monotone and Lipschitzian; namely, there are constants $\eta, L>0$ satisfying the properties

$$
\begin{gathered}
\langle\nabla f(x)-\nabla f(y), x-y\rangle \geq \eta\|x-y\|^{2}, \\
\|\nabla f(x)-\nabla f(y)\| \leq L\|x-y\|
\end{gathered}
$$

for all $x, y \in C$, then, for $0<\lambda<2 \eta / L^{2}$, the operator

$$
T:=P_{C}(I-\lambda \nabla f)
$$

is a contraction; hence, the sequence $\left\{x_{n}\right\}$ defined by algorithm (1.6) converges in norm to the unique solution of the minimization (1.5). More generally, if the sequence $\left\{\lambda_{n}\right\}$ is chosen to satisfy the property

$$
0<\liminf _{n \rightarrow \infty} \lambda_{n} \leq \limsup _{n \rightarrow \infty} \lambda_{n}<\frac{2 \eta}{L^{2}}
$$


then the sequence $\left\{x_{n}\right\}$ defined by algorithm (1.7) converges in norm to the unique minimizer of (1.5). However, if the gradient $\nabla f$ fails to be strongly monotone, the operator $T$ defined in (1.10) would fail to be contractive; consequently, the sequence $\left\{x_{n}\right\}$ generated by algorithm (1.6) may fail to converge strongly (see Section 4 in $\mathrm{Xu}$ [3]). The following theorem states that if the Lipschitz condition (1.9) holds, then the algorithms (1.6) and (1.7) can still converge in the weak topology.

Theorem 1.1 (see [3, Theorem 3.2]). Assume the minimization (1.5) is consistent and let $\Omega$ denote its solution set. Assume the gradient $\nabla f$ satisfies the Lipschitz condition (1.9). Let the sequence of parameters, $\left\{\lambda_{n}\right\}$, satisfy the condition

$$
0<\liminf _{n \rightarrow \infty} \leq \limsup _{n \rightarrow \infty} \lambda_{n}<\frac{2}{L}
$$

Then the sequence $\left\{x_{n}\right\}$ generated by the gradient-projection algorithm (1.7) converges weakly to a minimizer of (1.5).

From the above theorem, it is known that the gradient-projection algorithm has weak convergence, in general, unless the underlying Hilbert space is finite dimensional. This gives naturally rise to a question how to appropriately modify the gradient-projection algorithm so as to have strong convergence. $\mathrm{Xu}$ [3] gave two such modifications, one of which is simply a convex combination of a contraction with the point generated by the projected gradient algorithm.

Theorem 1.2 (see [3, Theorem 5.2]). Assume the minimization (1.5) is consistent and let $\Omega$ denote its solution set. Assume the gradient $\nabla f$ satisfies the Lipschitz condition (1.9). Let $Q: C \rightarrow C$ be a $\rho$-contraction with $\rho \in[0,1)$. Let a sequence $\left\{x_{n}\right\}$ be generated by the following hybrid gradientprojection algorithm:

$$
x_{n+1}=\alpha_{n} Q x_{n}+\left(1-\alpha_{n}\right) P_{C}\left(x_{n}-\lambda_{n} \nabla f\left(x_{n}\right)\right), \quad \forall n \geq 0 .
$$

Assume the sequence $\left\{\lambda_{n}\right\}$ satisfies the condition (1.12) and, in addition, the following conditions are satisfied for $\left\{\lambda_{n}\right\}$ and $\left\{\alpha_{n}\right\} \subset[0,1]$ :

(i) $\alpha_{n} \rightarrow 0$;

(ii) $\sum_{n=0}^{\infty} \alpha_{n}=\infty$;

(iii) $\sum_{n=0}^{\infty}\left|\alpha_{n+1}-\alpha_{n}\right|<\infty$;

(iv) $\sum_{n=0}^{\infty}\left|\lambda_{n+1}-\lambda_{n}\right|<\infty$.

Then the sequence $\left\{x_{n}\right\}$ converges in norm to a minimizer of (1.5) which is also the unique solution of the variational inequality of finding $x^{*} \in \Omega$ such that

$$
\left\langle(I-Q) x^{*}, x-x^{*}\right\rangle \geq 0, \quad \forall x \in \Omega .
$$

In other words, $x^{*}$ is the unique fixed point of the contraction $P_{\Omega} Q, x^{*}=P_{\Omega} Q x^{*}$.

On the other hand, Peng and Yao [4] recently introduced the following generalized mixed equilibrium problem of finding $\bar{x} \in C$ such that

$$
\Theta(\bar{x}, y)+\varphi(y)-\varphi(\bar{x})+\langle F \bar{x}, y-\bar{x}\rangle \geq 0, \quad \forall y \in C,
$$


where $F: C \rightarrow H$ is a nonlinear mapping and $\varphi: C \rightarrow \mathbf{R}$ is a function and $\Theta: C \times C \rightarrow \mathbf{R}$ is a bifunction. The set of solutions of problem (1.15) is denoted by GMEP. Subsequently, Yao et al. [5] and Ceng and Yao [6] also considered problem (1.15).

The problem (1.15) is very general in the sense that it includes, as special cases, optimization problems, variational inequalities, minimax problems, Nash equilibrium problems in noncooperative games, and others; see, for example, [7-15]. Here some special cases of problem (1.15) are stated as follows.

If $F=0$, then problem (1.15) reduces to the following mixed equilibrium problem of finding $\bar{x} \in C$ such that

$$
\Theta(\bar{x}, y)+\varphi(y)-\varphi(\bar{x}) \geq 0, \quad \forall y \in C
$$

which was considered by Ceng and Yao [7] and Bigi et al. [16]. Very recently, Peng [10] further discussed this problem. The set of solutions of this problem is denoted by MEP.

If $\varphi=0$, then problem (1.15) reduces to the following generalized equilibrium problem of finding $\bar{x} \in C$ such that

$$
\Theta(\bar{x}, y)+\langle F \bar{x}, y-\bar{x}\rangle \geq 0, \quad \forall y \in C
$$

which was studied by S. Takahashi and W. Takahashi [8].

If $\varphi=0$ and $F=0$, then problem (1.15) reduces to the following equilibrium problem of finding $\bar{x} \in C$ such that

$$
\Theta(\bar{x}, y) \geq 0, \quad \forall y \in C
$$

If $\Theta=0, \varphi=0$ and $F=A$, then problem (1.15) reduces to the following classical variational inequality of finding $\bar{x} \in C$ such that

$$
\langle A \bar{x}, y-\bar{x}\rangle \geq 0, \quad \forall y \in C
$$

whose solution set is denoted by $\operatorname{VI}(C, A)$.

The variational inequalities have been extensively studied in the literature; see [14, 1727] and the references therein. In 2006, Nadezhkina and Takahashi [22, 25] and Zeng and Yao [18] proposed some variants of Korpelevič's extragradient method [17] for finding an element of $\operatorname{Fix}(S) \cap \operatorname{VI}(C, A)$, where $S$ is a nonexpansive self-mapping on $C$.

Very recently, Peng [10] also introduced a variant of Korpelevič's extragradient method [17] for finding a common element of the set of solutions of a mixed equilibrium problem, the set of fixed points of a strict pseudocontraction, and the set of solutions of a variational inequality for a monotone, Lipschitz continuous mapping.

Theorem 1.3 (see [10, Theorem 3.1]). Let $C$ be a nonempty closed convex subset of a real Hilbert space $H$. Let $\Theta: C \times C \rightarrow \mathbf{R}$ be a bifunction satisfying conditions (H1)-(H4) and $\varphi: C \rightarrow \mathbf{R} a$ lower semicontinuous and convex function with assumptions (A1) or (A2), where

(H1) $\Theta(x, x)=0$, for all $x \in C$;

(H2) $\Theta$ is monotone, that is, $\Theta(x, y)+\Theta(y, x) \leq 0$, for all $x, y \in C$; 
Journal of Function Spaces and Applications

(H3) for each $y \in C, x \mapsto \Theta(x, y)$ is weakly upper semicontinuous;

(H4) for each $x \in C, y \mapsto \Theta(x, y)$ is convex and lower semicontinuous;

(A1) for each $x \in H$ and $r>0$, there exists a bounded subset $D_{x} \subset C$ and $y_{x} \in C$ such that for any $z \in C \backslash D_{x}$

$$
\Theta\left(z, y_{x}\right)+\varphi\left(y_{x}\right)-\varphi(z)+\frac{1}{r}\left\langle y_{x}-z, z-x\right\rangle<0
$$

(A2) $C$ is a bounded set.

Let $A: C \rightarrow H$ be a monotone and L-Lipschitz-continuous mapping and $S: C \rightarrow C$ be a $k$-strict pseudocontraction for some $0 \leq k<1$ such that $\operatorname{Fix}(S) \cap \operatorname{VI}(C, A) \cap \mathrm{MEP} \neq \emptyset$. For given $x_{0} \in H$ arbitrarily, let $\left\{x_{n}\right\},\left\{t_{n}\right\},\left\{y_{n}\right\},\left\{u_{n}\right\},\left\{z_{n}\right\}$ be sequences generated by

$$
\begin{gathered}
\Theta\left(t_{n}, y\right)+\varphi(y)-\varphi\left(t_{n}\right)+\frac{1}{r_{n}}\left\langle y-t_{n}, t_{n}-x_{n}\right\rangle \geq 0, \quad \forall y \in C, \\
y_{n}=P_{C}\left(t_{n}-\lambda_{n} A t_{n}\right), \\
u_{n}=P_{C}\left(t_{n}-\lambda_{n} A y_{n}\right), \\
z_{n}=\alpha_{n} u_{n}+\left(1-\alpha_{n}\right) S u_{n}, \\
C_{n}=\left\{z \in C:\left\|z_{n}-z\right\|^{2} \leq\left\|x_{n}-z\right\|^{2}-\left(1-\alpha_{n}\right)\left(\alpha_{n}-\varepsilon\right)\left\|t_{n}-S t_{n}\right\|^{2}\right\}, \\
Q_{n}=\left\{z \in H:\left\langle x_{n}-z, x-x_{n}\right\rangle \geq 0\right\}, \\
x_{n+1}=P_{C_{n} \cap Q_{n}} x, \quad \forall n \geq 0 .
\end{gathered}
$$

Assume that $\left\{\lambda_{n}\right\} \subset[a, b]$ for some $a, b \in(0,1 / L),\left\{\alpha_{n}\right\} \subset[c, d]$ for some $c, d \in(k, 1)$ and let $\left\{r_{n}\right\} \subset(0, \infty)$ satisfy $\liminf _{n \rightarrow \infty} r_{n}>0$. Then, $\left\{x_{n}\right\},\left\{t_{n}\right\},\left\{y_{n}\right\},\left\{u_{n}\right\},\left\{z_{n}\right\}$ converge strongly to $w=P_{\mathrm{Fix}(S) \cap \mathrm{VI}(C, A) \cap \mathrm{MEP} x}$.

Furthermore, related iterative methods for solving fixed point problems, variational inequalities, equilibrium problems, and optimization problems can be found in $[1,2,6,11,13-$ $16,19,20,24,26-35]$.

In this paper, let $C$ be a nonempty closed convex subset of a real Hilbert space $H$. Let $\Theta: C \times C \rightarrow \mathbf{R}$ be a bifunction satisfying conditions $(\mathrm{H} 1)-(\mathrm{H} 4)$ and $\varphi: C \rightarrow \mathbf{R}$ a lower semicontinuous and convex function with assumptions (A1) or (A2). Suppose the minimization (1.5) is consistent and let $\Omega$ denote its solution set. Let the gradient $\nabla f$ be $L$-Lipschitzian with constant $L>0$ and $F: C \rightarrow H$ be an $\alpha$-inverse strongly monotone mapping. Let $S: C \rightarrow C$ be a $k$-strictly pseudocontractive mapping such that $\operatorname{Fix}(S) \cap \Omega \cap$ GMEP $\neq \emptyset$. Let $Q: C \rightarrow C$ be a $\rho$-contraction with $\rho \in[0,1 / 2)$. For given $x_{0} \in C$ arbitrarily, let the sequences $\left\{x_{n}\right\},\left\{y_{n}\right\}$ and $\left\{z_{n}\right\}$ be generated iteratively by

$$
\begin{gathered}
\Theta\left(z_{n}, y\right)+\varphi(y)-\varphi\left(z_{n}\right)+\left\langle F x_{n}, y-z_{n}\right\rangle+\frac{1}{r_{n}}\left\langle y-z_{n}, z_{n}-x_{n}\right\rangle \geq 0, \quad \forall y \in C, \\
y_{n}=\alpha_{n} Q x_{n}+\left(1-\alpha_{n}\right) P_{C}\left(z_{n}-\lambda_{n} \nabla f\left(z_{n}\right)\right), \\
x_{n+1}=\beta_{n} x_{n}+\gamma_{n} P_{C}\left(z_{n}-\lambda_{n} \nabla f\left(z_{n}\right)\right)+\delta_{n} S y_{n}, \quad \forall n \geq 0,
\end{gathered}
$$


where $\left\{\lambda_{n}\right\} \subset(0,2 / L],\left\{r_{n}\right\} \subset(0,2 \alpha]$ and $\left\{\alpha_{n}\right\},\left\{\beta_{n}\right\},\left\{\gamma_{n}\right\},\left\{\delta_{n}\right\}$ are four sequences in $[0,1]$ such that $\beta_{n}+\gamma_{n}+\delta_{n}=1$ for all $n \geq 0$. It is proven that under very mild conditions, the sequences $\left\{x_{n}\right\},\left\{y_{n}\right\}$ and $\left\{z_{n}\right\}$ converge strongly to the unique solution of the variational inequality of finding $x^{*} \in \operatorname{Fix}(S) \cap \Omega \cap$ GMEP such that

$$
\left\langle(I-Q) x^{*}, x-x^{*}\right\rangle \geq 0, \quad \forall x \in \operatorname{Fix}(S) \cap \Omega \cap \text { GMEP } .
$$

In other words, $x^{*}$ is the unique fixed point of the contraction $P_{\operatorname{Fix}(S) \cap \Omega \cap G M E P} Q, x^{*}=$ $P_{\text {Fix }(S) \cap \Omega \cap G M E P} Q x^{*}$. The result presented in this paper generalizes and improves some wellknown results in the literature. Indeed, compared with some well-known results in the literature, our result improves and extends them in the following aspects.

(i) Compared with $\mathrm{Xu}$ [3, Theorem 3.2], a weak convergence result, our result is a strong convergence result.

(ii) Our problem of finding an element of $\operatorname{Fix}(S) \cap \Omega \cap$ GMEP is more general than the problem of finding an element of $\operatorname{Fix}(S) \cap \operatorname{VI}(C, A)$ in $[14,18,22,23,25]$.

(iii) In our algorithm (1.22), Xu's modified gradient-projection algorithm in [3, Theorem 5.2] is rewritten as the second iteration step

$$
y_{n}=\alpha_{n} Q x_{n}+\left(1-\alpha_{n}\right) P_{C}\left(z_{n}-\lambda_{n} \nabla f\left(z_{n}\right)\right) .
$$

Here the main purpose of the reason why we use such an iteration step is to play a convenience and efficiency role in the computation of an element of $\Omega$. Therefore, Xu's algorithm (1.13) is extended to develop our algorithm (1.22).

(iv) Our problem of finding an element of $\operatorname{Fix}(S) \cap \Omega \cap$ GMEP is more general than the problem of finding an element of $\Omega$ in Xu [3]. In addition, it is worth pointing out that Xu's conditions $\sum_{n=0}^{\infty}\left|\alpha_{n+1}-\alpha_{n}\right|<\infty$ and $\sum_{n=0}^{\infty}\left|\lambda_{n+1}-\lambda_{n}\right|<\infty$ in the above Theorem 1.2 are replaced by the weaker conditions $\lim _{n \rightarrow \infty}\left(\alpha_{n}-\alpha_{n+1}\right)=0$ and $\lim _{n \rightarrow \infty}\left(\lambda_{n}-\lambda_{n+1}\right)=0$ in our result (see Theorem 3.2 in Section 3).

\section{Preliminaries}

Let $H$ be a real Hilbert space with inner product $\langle\cdot, \cdot\rangle$ and norm $\|\cdot\|$ and $C$ a nonempty closed convex subset of $H$. We write $\rightarrow$ to indicate that the sequence $\left\{x_{n}\right\}$ converges strongly to $x$ and $\rightarrow$ to indicate that the sequence $\left\{x_{n}\right\}$ converges weakly to $x$. Moreover, we use $\omega_{w}\left(x_{n}\right)$ to denote the weak $\omega$-limit set of the sequence $\left\{x_{n}\right\}$, that is,

$$
\omega_{w}\left(x_{n}\right):=\left\{x: x_{n_{i}}-x \text { for some subsequence }\left\{x_{n_{i}}\right\} \text { of }\left\{x_{n}\right\}\right\} .
$$

For every point $x \in H$, there exists a unique nearest point in $C$, denoted by $P_{C} x$, such that

$$
\left\|x-P_{C} x\right\| \leq\|x-y\|, \quad \forall x \in C .
$$

$P_{C}$ is called the metric projection of $H$ onto $C$. We know that $P_{C}$ is a firmly nonexpansive mapping of $H$ onto $C$; that is, there holds the following relation:

$$
\left\langle P_{C} x-P_{C} y, x-y\right\rangle \geq\left\|P_{C} x-P_{C} y\right\|^{2}, \quad \forall x, y \in H .
$$


Consequently, $P_{C}$ is nonexpansive and monotone. It is also known that $P_{C}$ is characterized by the following properties: $P_{C} x \in C$ and

$$
\begin{gathered}
\left\langle x-P_{C} x, P_{C} x-y\right\rangle \geq 0, \\
\|x-y\|^{2} \geq\left\|x-P_{C} x\right\|^{2}+\left\|y-P_{C} x\right\|^{2},
\end{gathered}
$$

for all $x \in H, y \in C$; see [36] for more details. Let $A: C \rightarrow H$ be a monotone mapping. In the context of the variational inequality, this implies that

$$
x \in \operatorname{VI}(C, A) \Longleftrightarrow x=P_{C}(x-\lambda A x) \quad \forall \lambda>0 .
$$

A set-valued mapping $T: H \rightarrow 2^{H}$ is called monotone if for all $x, y \in H, f \in T x$ and $g \in T y$ imply $\langle f-g, x-y\rangle \geq 0$. A monotone mapping $T: H \rightarrow 2^{H}$ is called maximal if its graph $G(T)$ is not properly contained in the graph of any other monotone mapping. It is known that a monotone mapping $T$ is maximal if and only if for $(x, f) \in H \times H,\langle f-g, x-y\rangle \geq$ 0 for every $(y, g) \in G(T)$ implies $f \in T x$.

Let $A: C \rightarrow H$ be a monotone, $k$-Lipschitz-continuous mapping and let $N_{C} v$ be the normal cone to $C$ at $v \in C$, that is, $N_{C} v=\{w \in H:\langle v-u, w\rangle \geq 0$, for all $u \in C\}$. Define

$$
T v= \begin{cases}A v+N_{C} v, & \text { if } v \in C \\ \emptyset, & \text { if } v \notin C\end{cases}
$$

Then, $T$ is maximal monotone and $0 \in T v$ if and only if $v \in \mathrm{VI}(C, A)$; see [37].

Recall that a mapping $S: C \rightarrow C$ is called a strict pseudocontraction if there exists a constant $0 \leq k<1$ such that

$$
\|S x-S y\|^{2} \leq\|x-y\|^{2}+k\|(I-S) x-(I-S) y\|^{2}, \quad \forall x, y \in C
$$

In this case, we also say that $S$ is a $k$-strict pseudocontraction. A mapping $A: C \rightarrow H$ is called $\alpha$-inverse strongly monotone if there exists a constant $\alpha>0$ such that

$$
\langle A x-A y, x-y\rangle \geq \alpha\|A x-A y\|^{2}, \quad \forall x, y \in C .
$$

It is obvious that any $\alpha$-inverse strongly monotone mapping is Lipschitz continuous. Meantime, observe that (2.8) is equivalent to

$$
\langle S x-S y, x-y\rangle \leq\|x-y\|^{2}-\frac{1-k}{2}\|(I-S) x-(I-S) y\|^{2}, \quad \forall x, y \in C .
$$

It is easy to see that if $S$ is a $k$-strictly pseudocontractive mapping, then $I-S$ is $((1-k) / 2)$ inverse strongly monotone and hence $(2 /(1-k))$-Lipschitz continuous. Thus, $S$ is Lipschitz continuous with constant $(1+k) /(1-k)$. We denote by Fix $(S)$ the set of fixed points of $S$. It is clear that the class of strict pseudocontractions strictly includes the one of nonexpansive mappings which are mappings $S: C \rightarrow C$ such that $\|S x-S y\| \leq\|x-y\|$ for all $x, y \in C$. 
In order to prove our main result in the next section, we need the following lemmas and propositions.

Lemma 2.1 (see [7]). Let $C$ be a nonempty closed convex subset of a real Hilbert space $H$. Let $\Theta: C \times$ $\mathrm{C} \rightarrow \mathbf{R}$ be a bifunction satisfying conditions (H1)-(H4) and let $\varphi: C \rightarrow \mathbf{R}$ be a lower semicontinuous and convex function. For $r>0$ and $x \in H$, define a mapping $T_{r}^{(\Theta, \varphi)}: H \rightarrow C$ as follows:

$$
T_{r}^{(\Theta, \varphi)}(x)=\left\{z \in C: \Theta(z, y)+\varphi(y)-\varphi(z)+\frac{1}{r}\langle y-z, z-x\rangle \geq 0, \forall y \in C\right\}
$$

for all $x \in H$. Assume that either (A1) or (A2) holds. Then the following conclusions hold:

(i) $T_{r}^{(\Theta, \varphi)}(x) \neq \emptyset$ for each $x \in H$ and $T_{r}^{(\Theta, \varphi)}$ is single-valued;

(ii) $T_{r}^{(\Theta, \varphi)}$ is firmly nonexpansive, that is, for any $x, y \in H$,

$$
\left\|T_{r}^{(\Theta, \varphi)} x-T_{r}^{(\Theta, \varphi)} y\right\|^{2} \leq\left\langle T_{r}^{(\Theta, \varphi)} x-T_{r}^{(\Theta, \varphi)} y, x-y\right\rangle
$$

(iii) $\operatorname{Fix}\left(T_{r}^{(\Theta, \varphi)}\right)=\operatorname{MEP}(\Theta, \varphi)$;

(iv) $\operatorname{MEP}(\Theta, \varphi)$ is closed and convex.

Remark 2.2. If $\varphi=0$, then $T_{r}^{(\Theta, \varphi)}$ is rewritten as $T_{r}^{\Theta}$.

The following lemma is an immediate consequence of an inner product.

Lemma 2.3. In a real Hilbert space $H$, there holds the inequality

$$
\|x+y\|^{2} \leq\|x\|^{2}+2\langle y, x+y\rangle, \quad \forall x, y \in H
$$

Proposition 2.4 (see [6, Proposition 2.1]). Let $C, H, \Theta, \varphi$, and $T_{r}^{(\Theta, \varphi)}$ be as in Lemma 2.1. Then the following relation holds:

$$
\left\|T_{s}^{(\Theta, \varphi)} x-T_{t}^{(\Theta, \varphi)} x\right\|^{2} \leq \frac{s-t}{s}\left\langle T_{s}^{(\Theta, \varphi)} x-T_{t}^{(\Theta, \varphi)} x, T_{s}^{(\Theta, \varphi)} x-x\right\rangle
$$

for all $s, t>0$ and $x \in H$.

Recall that $S: C \rightarrow C$ is called a quasi-strict pseudocontraction if the fixed point set of $S, \operatorname{Fix}(S)$, is nonempty and if there exists a constant $0 \leq k<1$ such that

$$
\|S x-p\|^{2} \leq\|x-p\|^{2}+k\|x-S x\|^{2} \quad \forall x \in C, p \in \operatorname{Fix}(S) .
$$

We also say that $S$ is a $k$-quasi-strict pseudocontraction if condition (2.15) holds.

Proposition 2.5 (see [2, Proposition 2.1]). Assume $C$ is a nonempty closed convex subset of a real Hilbert space $H$ and let $S: C \rightarrow C$ be a self-mapping on $C$. 
Journal of Function Spaces and Applications

(i) If $S$ is a $k$-strict pseudocontraction, then $S$ satisfies the Lipschitz condition

$$
\|S x-S y\| \leq \frac{1+k}{1-k}\|x-y\|, \quad \forall x, y \in C
$$

(ii) If $S$ is a $k$-strict pseudocontraction, then the mapping $I-S$ is demiclosed (at 0$)$. That is, if $\left\{x_{n}\right\}$ is a sequence in $C$ such that $x_{n}-\tilde{x}$ and $(I-S) x_{n} \rightarrow 0$, then $(I-S) \tilde{x}=0$, that is, $\tilde{x} \in \operatorname{Fix}(S)$.

(iii) If $S$ is a $k$-quasi-strict pseudocontraction, then the fixed point set Fix $(S)$ of $S$ is closed and convex so that the projection $P_{\mathrm{Fix}(S)}$ is well defined.

The following lemma was proved by Suzuki [30].

Lemma 2.6 (see [30]). Let $\left\{x_{n}\right\}$ and $\left\{y_{n}\right\}$ be bounded sequences in a Banach space $X$ and let $\left\{\beta_{n}\right\}$ be a sequence in $[0,1]$ with $0<\liminf _{n \rightarrow \infty} \beta_{n} \leq \limsup _{n \rightarrow \infty} \beta_{n}<1$. Suppose $x_{n+1}=\left(1-\beta_{n}\right) y_{n}+\beta_{n} x_{n}$ for all integers $n \geq 0$ and $\lim \sup _{n \rightarrow \infty}\left(\left\|y_{n+1}-y_{n}\right\|-\left\|x_{n+1}-x_{n}\right\|\right) \leq 0$. Then, $\lim _{n \rightarrow \infty}\left\|y_{n}-x_{n}\right\|=0$.

Lemma 2.7 (see [34]). Let $\left\{a_{n}\right\}$ be a sequence of nonnegative numbers satisfying the condition

$$
a_{n+1} \leq\left(1-\delta_{n}\right) a_{n}+\delta_{n} \sigma_{n}, \quad \forall n \geq 0,
$$

where $\left\{\delta_{n}\right\},\left\{\sigma_{n}\right\}$ are sequences of real numbers such that

(i) $\left\{\delta_{n}\right\} \subset[0,1]$ and $\sum_{n=0}^{\infty} \delta_{n}=\infty$, or equivalently,

$$
\prod_{n=0}^{\infty}\left(1-\delta_{n}\right):=\lim _{n \rightarrow \infty} \prod_{j=0}^{n}\left(1-\delta_{j}\right)=0
$$

(ii) $\limsup _{n \rightarrow \infty} \sigma_{n} \leq 0$, or,

(iii) $\sum_{n=0}^{\infty} \delta_{n} \sigma_{n}$ is convergent.

Then $\lim _{n \rightarrow \infty} a_{n}=0$.

\section{Strong Convergence Theorem}

In order to prove our main result, we shall need the following lemma given in [21].

Lemma 3.1. Let $C$ be a nonempty closed convex subset of a real Hilbert space $H$. Let $S: C \rightarrow C$ be a $k$-strictly pseudocontractive mapping. Let $\gamma$ and $\delta$ be two nonnegative real numbers. Assume $(\gamma+\delta) k \leq r$. Then

$$
\|\gamma(x-y)+\delta(S x-S y)\| \leq(\gamma+\delta)\|x-y\|, \quad \forall x \cdot y \in C
$$

We are now in a position to state and prove our main result.

Theorem 3.2. Let $C$ be a nonempty bounded closed convex subset of a real Hilbert space $H$. Let $\Theta$ : $\mathrm{C} \times \mathrm{C} \rightarrow \mathbf{R}$ be a bifunction satisfying conditions (H1)-(H4) and $\varphi: C \rightarrow \mathbf{R}$ a lower semicontinuous 
and convex function with assumptions (A1) or (A2). Suppose the minimization (1.5) is consistent and let $\Omega$ denote its solution set. Assume the gradient $\nabla f$ is L-Lipschitzian with constant $L>0$ and $F: C \rightarrow H$ is an $\alpha$-inverse strongly monotone mapping. Let $S: C \rightarrow C$ be a k-strictly pseudocontractive mapping such that $\operatorname{Fix}(S) \cap \Omega \cap \mathrm{GMEP} \neq \emptyset$. Let $Q: C \rightarrow C$ be a $\rho$-contraction with $\rho \in[0,1 / 2)$. For given $x_{0} \in C$ arbitrarily, let the sequences $\left\{x_{n}\right\},\left\{y_{n}\right\}$, and $\left\{z_{n}\right\}$ be generated iteratively by

$$
\begin{gathered}
\Theta\left(z_{n}, y\right)+\varphi(y)-\varphi\left(z_{n}\right)+\left\langle F x_{n}, y-z_{n}\right\rangle+\frac{1}{r_{n}}\left\langle y-z_{n}, z_{n}-x_{n}\right\rangle \geq 0, \quad \forall y \in C, \\
y_{n}=\alpha_{n} Q x_{n}+\left(1-\alpha_{n}\right) P_{C}\left(z_{n}-\lambda_{n} \nabla f\left(z_{n}\right)\right) \\
x_{n+1}=\beta_{n} x_{n}+\gamma_{n} P_{C}\left(z_{n}-\lambda_{n} \nabla f\left(z_{n}\right)\right)+\delta_{n} S y_{n}, \quad \forall n \geq 0,
\end{gathered}
$$

where $\left\{\lambda_{n}\right\} \subset(0,2 / L],\left\{r_{n}\right\} \subset(0,2 \alpha]$, and $\left\{\alpha_{n}\right\},\left\{\beta_{n}\right\},\left\{\gamma_{n}\right\},\left\{\delta_{n}\right\}$ are four sequences in $[0,1]$ such that

(i) $0<\liminf _{n \rightarrow \infty} \lambda_{n} \leq \limsup \sup _{n \rightarrow \infty} \lambda_{n}<2 / L$ and $\lim _{n \rightarrow \infty}\left(\lambda_{n}-\lambda_{n+1}\right)=0$;

(ii) $0<\liminf _{n \rightarrow \infty} r_{n} \leq \limsup _{n \rightarrow \infty} r_{n}<2 \alpha$ and $\lim _{n \rightarrow \infty}\left(r_{n}-r_{n+1}\right)=0$;

(iii) $\beta_{n}+\gamma_{n}+\delta_{n}=1$ and $\left(\gamma_{n}+\delta_{n}\right) k \leq \gamma_{n}<(1-2 \rho) \delta_{n}$ for all $n \geq 0$;

(iv) $\lim _{n \rightarrow \infty} \alpha_{n}=0$ and $\sum_{n=0}^{\infty} \alpha_{n}=\infty$;

(v) $0<\liminf _{n \rightarrow \infty} \beta_{n} \leq \lim \sup _{n \rightarrow \infty} \beta_{n}<1$ and $\liminf _{n \rightarrow \infty} \delta_{n}>0$;

(vi) $\lim _{n \rightarrow \infty}\left(\gamma_{n+1} /\left(1-\beta_{n+1}\right)-\gamma_{n} /\left(1-\beta_{n}\right)\right)=0$.

Then the sequences $\left\{x_{n}\right\},\left\{y_{n}\right\}$, and $\left\{z_{n}\right\}$ converge strongly to the unique solution of the variational inequality of finding $x^{*} \in \operatorname{Fix}(S) \cap \Omega \cap$ GMEP such that

$$
\left\langle(I-Q) x^{*}, x-x^{*}\right\rangle \geq 0, \quad \forall x \in \operatorname{Fix}(S) \cap \Omega \cap \text { GMEP } .
$$

In other words, $x^{*}$ is the unique fixed point of the contraction $P_{\operatorname{Fix}(S) \cap \Omega \cap G M E P} Q, x^{*}=$ $P_{\text {Fix }(S) \cap \Omega \cap G M E P} Q x^{*}$.

Proof. First it is obvious that there hold the following assertions:

(a) $x^{*} \in C$ solves the minimization (1.5);

(b) $x^{*}$ solves the fixed point equation

$$
x^{*}=P_{C}(I-\lambda \nabla f) x^{*},
$$

where $\lambda>0$ is any fixed positive number;

(c) $x^{*}$ solves the variational inequality of finding $x^{*} \in C$ such that

$$
\left\langle\nabla f\left(x^{*}\right), x-x^{*}\right\rangle \geq 0, \quad \forall x \in C,
$$

where its solution set is denoted by $\operatorname{VI}(C, \nabla f)$.

We divide the proof into several steps.

Step 1 . We claim that $\lim _{n \rightarrow \infty}\left\|x_{n+1}-x_{n}\right\|=0$. 
Journal of Function Spaces and Applications

Indeed, first, we can write (3.2) as $x_{n+1}=\beta_{n} x_{n}+\left(1-\beta_{n}\right) u_{n}$, for all $n \geq 0$, where $u_{n}=$ $\left(x_{n+1}-\beta_{n} x_{n}\right) /\left(1-\beta_{n}\right)$. It follows that

$$
\begin{aligned}
u_{n+1}-u_{n}= & \frac{x_{n+2}-\beta_{n+1} x_{n+1}}{1-\beta_{n+1}}-\frac{x_{n+1}-\beta_{n} x_{n}}{1-\beta_{n}} \\
= & \frac{\gamma_{n+1} P_{C}\left(z_{n+1}-\lambda_{n+1} \nabla f\left(z_{n+1}\right)\right)+\delta_{n+1} S y_{n+1}}{1-\beta_{n+1}}-\frac{\gamma_{n} P_{C}\left(z_{n}-\lambda_{n} \nabla f\left(z_{n}\right)\right)+\delta_{n} S y_{n}}{1-\beta_{n}} \\
= & \frac{\gamma_{n+1}\left[P_{C}\left(z_{n+1}-\lambda_{n+1} \nabla f\left(z_{n+1}\right)\right)-P_{C}\left(z_{n}-\lambda_{n} \nabla f\left(z_{n}\right)\right)\right]+\delta_{n+1}\left(S y_{n+1}-S y_{n}\right)}{1-\beta_{n+1}} \\
& +\left(\frac{\gamma_{n+1}}{1-\beta_{n+1}}-\frac{\gamma_{n}}{1-\beta_{n}}\right) P_{C}\left(z_{n}-\lambda_{n} \nabla f\left(z_{n}\right)\right)+\left(\frac{\delta_{n+1}}{1-\beta_{n+1}}-\frac{\delta_{n}}{1-\beta_{n}}\right) S y_{n} .
\end{aligned}
$$

From Lemma 3.1 and (3.2), we get

$$
\begin{aligned}
\| \gamma_{n+1}[ & \left.P_{C}\left(z_{n+1}-\lambda_{n+1} \nabla f\left(z_{n+1}\right)\right)-P_{C}\left(z_{n}-\lambda_{n} \nabla f\left(z_{n}\right)\right)\right]+\delta_{n+1}\left(S y_{n+1}-S y_{n}\right) \| \\
\leq & \left\|\gamma_{n+1}\left(y_{n+1}-y_{n}\right)+\delta_{n+1}\left(S y_{n+1}-S y_{n}\right)\right\| \\
& +\gamma_{n+1}\left\|\left[P_{C}\left(z_{n+1}-\lambda_{n+1} \nabla f\left(z_{n+1}\right)\right)-y_{n+1}\right]+\left[y_{n}-P_{C}\left(z_{n}-\lambda_{n} \nabla f\left(z_{n}\right)\right)\right]\right\| \\
\leq & \left(\gamma_{n+1}+\delta_{n+1}\right)\left\|y_{n+1}-y_{n}\right\|+\gamma_{n+1} \alpha_{n+1}\left\|Q x_{n+1}-P_{C}\left(z_{n+1}-\lambda_{n+1} \nabla f\left(z_{n+1}\right)\right)\right\| \\
& +\gamma_{n+1} \alpha_{n}\left\|Q x_{n}-P_{C}\left(z_{n}-\lambda_{n} \nabla f\left(z_{n}\right)\right)\right\| .
\end{aligned}
$$

Let $\left\{T_{r_{n}}^{(\Theta, \varphi)}\right\}$ be a sequence of mappings defined as in Lemma 2.1. Note that the L-Lipschitz continuity of $\nabla f$ implies that the gradient $\nabla f$ is $(1 / L)$-ism [31]. Since $\nabla f$ and $F$ are $(1 / L)$ inverse strongly monotone mapping and $\alpha$-inverse strongly monotone mapping, respectively, then we have

$$
\begin{aligned}
& \|(I-\lambda \nabla f) x-(I-\lambda \nabla f) y\|^{2} \\
& \quad=\|x-y\|^{2}-2 \lambda\langle\nabla f(x)-\nabla f(y), x-y\rangle+\lambda^{2}\|\nabla f(x)-\nabla f(y)\|^{2} \\
& \quad \leq\|x-y\|^{2}+\lambda\left(\lambda-\frac{2}{L}\right)\|\nabla f(x)-\nabla f(y)\|^{2}, \\
& \|(I-\mu F) x-(I-\mu F) y\|^{2} \leq\|x-y\|^{2}+\mu(\mu-2 \alpha)\|F x-F y\|^{2} .
\end{aligned}
$$

It is clear that if $0 \leq \lambda \leq 2 / L$ and $0 \leq \mu \leq 2 \alpha$, then $(I-\lambda \nabla f)$ and $(I-\mu F)$ are nonexpansive. It follows from that

$$
\begin{aligned}
& \left\|P_{C}\left(z_{n+1}-\lambda_{n+1} \nabla f\left(z_{n+1}\right)\right)-P_{C}\left(z_{n}-\lambda_{n} \nabla f\left(z_{n}\right)\right)\right\| \\
& \quad \leq\left\|z_{n+1}-\lambda_{n+1} \nabla f\left(z_{n+1}\right)-\left(z_{n}-\lambda_{n} \nabla f\left(z_{n}\right)\right)\right\| \\
& \quad \leq\left\|z_{n+1}-\lambda_{n+1} \nabla f\left(z_{n+1}\right)-\left(z_{n}-\lambda_{n+1} \nabla f\left(z_{n}\right)\right)\right\|+\left|\lambda_{n+1}-\lambda_{n}\right|\left\|\nabla f\left(z_{n}\right)\right\|
\end{aligned}
$$




$$
\begin{aligned}
\leq & \left\|z_{n+1}-z_{n}\right\|+\left|\lambda_{n+1}-\lambda_{n}\right|\left\|\nabla f\left(z_{n}\right)\right\| \\
= & \left\|T_{r_{n+1}}^{(\Theta, \varphi)}\left(x_{n+1}-r_{n+1} F x_{n+1}\right)-T_{r_{n}}^{(\Theta, \varphi)}\left(x_{n}-r_{n} F x_{n}\right)\right\|+\left|\lambda_{n+1}-\lambda_{n}\right|\left\|\nabla f\left(z_{n}\right)\right\| \\
\leq & \left\|T_{r_{n+1}}^{(\Theta, \varphi)}\left(x_{n+1}-r_{n+1} F x_{n+1}\right)-T_{r_{n+1}}^{(\Theta, \varphi)}\left(x_{n}-r_{n} F x_{n}\right)\right\| \\
& \left.+\| T_{r_{n+1}^{(\Theta, \varphi)}}^{(\Theta} x_{n}-r_{n} F x_{n}\right)-T_{r_{n}}^{(\Theta, \varphi)}\left(x_{n}-r_{n} F x_{n}\right)\left\|+\left|\lambda_{n+1}-\lambda_{n}\right|\right\| \nabla f\left(z_{n}\right) \| \\
\leq & \left\|\left(x_{n+1}-r_{n+1} F x_{n+1}\right)-\left(x_{n}-r_{n} F x_{n}\right)\right\| \\
& +\left\|T_{r_{n+1}}^{(\Theta, \varphi)}\left(x_{n}-r_{n} F x_{n}\right)-T_{r_{n}}^{(\Theta, \varphi)}\left(x_{n}-r_{n} F x_{n}\right)\right\|+\left|\lambda_{n+1}-\lambda_{n}\right|\left\|\nabla f\left(z_{n}\right)\right\| \\
\leq & \left\|\left(x_{n+1}-r_{n+1} F x_{n+1}\right)-\left(x_{n}-r_{n+1} F x_{n}\right)\right\|+\left|r_{n+1}-r_{n}\right|\left\|F x_{n}\right\| \\
& +\left\|T_{r_{n+1}}^{(\Theta, \varphi)}\left(x_{n}-r_{n} F x_{n}\right)-T_{r_{n}}^{(\Theta, \varphi)}\left(x_{n}-r_{n} F x_{n}\right)\right\|+\left|\lambda_{n+1}-\lambda_{n}\right|\left\|\nabla f\left(z_{n}\right)\right\| \\
\leq & \left\|x_{n+1}-x_{n}\right\|+\left\|T_{r_{n+1}}^{(\Theta, \varphi)}\left(x_{n}-r_{n} F x_{n}\right)-T_{r_{n}}^{(\Theta, \varphi)}\left(x_{n}-r_{n} F x_{n}\right)\right\| \\
& +\left|r_{n+1}-r_{n}\left\|F x_{n}\right\|+\right| \lambda_{n+1}-\lambda_{n} \mid\left\|\nabla f\left(z_{n}\right)\right\| .
\end{aligned}
$$

Then,

$$
\begin{aligned}
&\left\|y_{n+1}-y_{n}\right\| \\
& \leq\left\|P_{C}\left(z_{n+1}-\lambda_{n+1} \nabla f\left(z_{n+1}\right)\right)-P_{C}\left(z_{n}-\lambda_{n} \nabla f\left(z_{n}\right)\right)\right\| \\
&+\alpha_{n+1}\left\|Q x_{n+1}-P_{C}\left(z_{n+1}-\lambda_{n+1} \nabla f\left(z_{n+1}\right)\right)\right\|+\alpha_{n}\left\|Q x_{n}-P_{C}\left(z_{n}-\lambda_{n} \nabla f\left(z_{n}\right)\right)\right\| \\
& \leq\left\|x_{n+1}-x_{n}\right\|+\left\|T_{r_{n+1}}^{(\Theta, \varphi)}\left(x_{n}-r_{n} F x_{n}\right)-T_{r_{n}}^{(\Theta, \varphi)}\left(x_{n}-r_{n} F x_{n}\right)\right\| \\
&+\left|r_{n+1}-r_{n}\right|\left\|F x_{n}\right\|+\left|\lambda_{n+1}-\lambda_{n}\right|\left\|\nabla f\left(z_{n}\right)\right\| \\
&+\alpha_{n}\left\|Q x_{n}-P_{C}\left(z_{n}-\lambda_{n} \nabla f\left(z_{n}\right)\right)\right\|+\alpha_{n+1}\left\|Q x_{n+1}-P_{C}\left(z_{n+1}-\lambda_{n+1} \nabla f\left(z_{n+1}\right)\right)\right\| .
\end{aligned}
$$

So, from (3.6), (3.7), and (3.10), we have

$$
\begin{aligned}
\left\|u_{n+1}-u_{n}\right\| \leq & \left\|x_{n+1}-x_{n}\right\|+\left(1+\frac{\gamma_{n+1}}{1-\beta_{n+1}}\right) \alpha_{n}\left\|Q x_{n}-P_{C}\left(z_{n}-\lambda_{n} \nabla f\left(z_{n}\right)\right)\right\| \\
& +\left(1+\frac{\gamma_{n+1}}{1-\beta_{n+1}}\right) \alpha_{n+1}\left\|Q x_{n+1}-P_{C}\left(z_{n+1}-\lambda_{n+1} \nabla f\left(z_{n+1}\right)\right)\right\| \\
& +\left|\frac{\gamma_{n+1}}{1-\beta_{n+1}}-\frac{\gamma_{n}}{1-\beta_{n}}\right|\left(\left\|P_{C}\left(z_{n}-\lambda_{n} \nabla f\left(z_{n}\right)\right)\right\|+\left\|S y_{n}\right\|\right) \\
& +\left\|T_{r_{n+1}}^{(\Theta, \varphi)}\left(x_{n}-r_{n} F x_{n}\right)-T_{r_{n}}^{(\Theta, \varphi)}\left(x_{n}-r_{n} F x_{n}\right)\right\| \\
& +\left|r_{n+1}-r_{n}\right|\left\|F x_{n}\right\|+\left|\lambda_{n+1}-\lambda_{n}\right|\left\|\nabla f\left(z_{n}\right)\right\| .
\end{aligned}
$$


Journal of Function Spaces and Applications

Utilizing Proposition 2.4 and condition (ii), we have

$$
\lim _{n \rightarrow \infty}\left\|T_{r_{n+1}}^{(\Theta, \varphi)}\left(x_{n}-r_{n} F x_{n}\right)-T_{r_{n}}^{(\Theta, \varphi)}\left(x_{n}-r_{n} F x_{n}\right)\right\|=0 .
$$

This implies that

$$
\limsup _{n \rightarrow \infty}\left(\left\|u_{n+1}-u_{n}\right\|-\left\|x_{n+1}-x_{n}\right\|\right) \leq 0 .
$$

Hence by Lemma 2.6, we get $\lim _{n \rightarrow \infty}\left\|u_{n}-x_{n}\right\|=0$. Consequently,

$$
\lim _{n \rightarrow \infty}\left\|x_{n+1}-x_{n}\right\|=\lim _{n \rightarrow \infty}\left(1-\beta_{n}\right)\left\|u_{n}-x_{n}\right\|=0
$$

Step 2. We claim that $\lim _{n \rightarrow \infty}\left\|\nabla f\left(z_{n}\right)-\nabla f\left(x^{*}\right)\right\|=0$ and $\lim _{n \rightarrow \infty}\left\|F x_{n}-F x^{*}\right\|=0$.

Indeed, let $x^{*} \in \operatorname{Fix}(S) \cap \Omega \cap$ GMEP. Then we have $x^{*}=S x^{*}, x^{*}=P_{C}\left(x^{*}-\lambda_{n} \nabla f\left(x^{*}\right)\right)$ and

$$
x^{*}=T_{r_{n}}^{(\Theta, \varphi)}\left(x^{*}-r_{n} F x^{*}\right) .
$$

Hence from (3.8), we have

$$
\begin{aligned}
\left\|P_{C}\left(z_{n}-\lambda_{n} \nabla f\left(z_{n}\right)\right)-P_{C}\left(x^{*}-\lambda_{n} \nabla f\left(x^{*}\right)\right)\right\|^{2} & \leq\left\|\left(z_{n}-\lambda_{n} \nabla f\left(z_{n}\right)\right)-\left(x^{*}-\lambda_{n} \nabla f\left(x^{*}\right)\right)\right\|^{2} \\
& \leq\left\|z_{n}-x^{*}\right\|^{2}+\lambda_{n}\left(\lambda_{n}-\frac{2}{L}\right)\left\|\nabla f\left(z_{n}\right)-\nabla f\left(x^{*}\right)\right\|^{2}, \\
\left\|z_{n}-x^{*}\right\|^{2} & =\left\|T_{r_{n}}^{(\Theta, \varphi)}\left(x_{n}-r_{n} F x_{n}\right)-T_{r_{n}}^{(\Theta, \varphi)}\left(x^{*}-r_{n} F x^{*}\right)\right\|^{2} \\
& \leq\left\|\left(x_{n}-r_{n} F x_{n}\right)-\left(x^{*}-r_{n} F x^{*}\right)\right\|^{2} \\
& \leq\left\|x_{n}-x^{*}\right\|^{2}+r_{n}\left(r_{n}-2 \alpha\right)\left\|F x_{n}-F x^{*}\right\|^{2} .
\end{aligned}
$$

It follows from (3.2), (3.16), and (3.17) that

$$
\begin{aligned}
\left\|y_{n}-x^{*}\right\|^{2} \leq & \left(1-\alpha_{n}\right)\left\|P_{C}\left(z_{n}-\lambda_{n} \nabla f\left(z_{n}\right)\right)-P_{C}\left(x^{*}-\lambda_{n} \nabla f\left(x^{*}\right)\right)\right\|^{2}+\alpha_{n}\left\|Q x_{n}-x^{*}\right\|^{2} \\
\leq & \alpha_{n}\left\|Q x_{n}-x^{*}\right\|^{2}+\left\|z_{n}-x^{*}\right\|^{2}+\lambda_{n}\left(\lambda_{n}-\frac{2}{L}\right)\left\|\nabla f\left(z_{n}\right)-\nabla f\left(x^{*}\right)\right\|^{2} \\
\leq & \alpha_{n}\left\|Q x_{n}-x^{*}\right\|^{2}+\left\|x_{n}-x^{*}\right\|^{2}+r_{n}\left(r_{n}-2 \alpha\right)\left\|F x_{n}-F x^{*}\right\|^{2} \\
& +\lambda_{n}\left(\lambda_{n}-\frac{2}{L}\right)\left\|\nabla f\left(z_{n}\right)-\nabla f\left(x^{*}\right)\right\|^{2} .
\end{aligned}
$$


Utilizing the convexity of $\|\cdot\|$, we have

$$
\begin{aligned}
& \left\|x_{n+1}-x^{*}\right\|^{2} \\
& \quad=\left\|\beta_{n}\left(x_{n}-x^{*}\right)+\left(1-\beta_{n}\right) \frac{1}{1-\beta_{n}}\left[\gamma_{n}\left(P_{C}\left(z_{n}-\lambda_{n} \nabla f\left(z_{n}\right)\right)-x^{*}\right)+\delta_{n}\left(S y_{n}-x^{*}\right)\right]\right\|^{2} \\
& \leq \beta_{n}\left\|x_{n}-x^{*}\right\|^{2}+\left(1-\beta_{n}\right)\left\|\frac{\gamma_{n}}{1-\beta_{n}}\left(P_{C}\left(z_{n}-\lambda_{n} \nabla f\left(z_{n}\right)\right)-x^{*}\right)+\frac{\delta_{n}}{1-\beta_{n}}\left(S y_{n}-x^{*}\right)\right\|^{2} \\
& \quad=\beta_{n}\left\|x_{n}-x^{*}\right\|^{2}+\left(1-\beta_{n}\right)\left\|\frac{\gamma_{n}\left(y_{n}-x^{*}\right)+\delta_{n}\left(S y_{n}-x^{*}\right)}{1-\beta_{n}}+\frac{\alpha_{n} \gamma_{n}}{1-\beta_{n}}\left(P_{C}\left(z_{n}-\lambda_{n} \nabla f\left(z_{n}\right)\right)-Q x_{n}\right)\right\|^{2} \\
& \quad \leq \beta_{n}\left\|x_{n}-x^{*}\right\|^{2}+\left(1-\beta_{n}\right)\left\|\frac{\gamma_{n}\left(y_{n}-x^{*}\right)+\delta_{n}\left(S y_{n}-x^{*}\right)}{1-\beta_{n}}\right\|^{2}+M \alpha_{n} \\
& \leq \beta_{n}\left\|x_{n}-x^{*}\right\|^{2}+\left(1-\beta_{n}\right)\left\|y_{n}-x^{*}\right\|^{2}+M \alpha_{n},
\end{aligned}
$$

where $M>0$ is some appropriate constant. So, from (3.18) and (3.19), it follows that

$$
\begin{aligned}
\left\|x_{n+1}-x^{*}\right\|^{2} \leq & \left\|x_{n}-x^{*}\right\|^{2}+r_{n}\left(r_{n}-2 \alpha\right)\left(1-\beta_{n}\right)\left\|F x_{n}-F x^{*}\right\|^{2} \\
& +\lambda_{n}\left(\lambda_{n}-\frac{2}{L}\right)\left(1-\beta_{n}\right)\left\|\nabla f\left(z_{n}\right)-\nabla f\left(x^{*}\right)\right\|^{2}+\left(M+\left\|Q x_{n}-x^{*}\right\|^{2}\right) \alpha_{n} .
\end{aligned}
$$

Therefore,

$$
\begin{aligned}
& \lambda_{n}\left(\frac{2}{L}-\lambda_{n}\right)\left(1-\beta_{n}\right)\left\|\nabla f\left(z_{n}\right)-\nabla f\left(x^{*}\right)\right\|^{2}+r_{n}\left(2 \alpha-r_{n}\right)\left(1-\beta_{n}\right)\left\|F x_{n}-F x^{*}\right\|^{2} \\
& \quad \leq\left\|x_{n}-x^{*}\right\|^{2}-\left\|x_{n+1}-x^{*}\right\|^{2}+\left(M+\left\|Q x_{n}-x^{*}\right\|^{2}\right) \alpha_{n} \\
& \quad \leq\left(\left\|x_{n}-x^{*}\right\|+\left\|x_{n+1}-x^{*}\right\|\right)\left\|x_{n}-x_{n+1}\right\|+\left(M+\left\|Q x_{n}-x^{*}\right\|^{2}\right) \alpha_{n} .
\end{aligned}
$$

Since $\liminf _{n \rightarrow \infty} \lambda_{n}\left(2 / L-\lambda_{n}\right)\left(1-\beta_{n}\right)>0, \liminf _{n \rightarrow \infty} r_{n}\left(2 \alpha-r_{n}\right)\left(1-\beta_{n}\right)>0,\left\|x_{n}-x_{n+1}\right\| \rightarrow 0$ and $\alpha_{n} \rightarrow 0$, we have

$$
\lim _{n \rightarrow \infty}\left\|\nabla f\left(z_{n}\right)-\nabla f\left(x^{*}\right)\right\|=0, \quad \lim _{n \rightarrow \infty}\left\|F x_{n}-F x^{*}\right\|=0 .
$$

Step 3. We claim that $\lim _{n \rightarrow \infty}\left\|S y_{n}-y_{n}\right\|=0$. 

have

Indeed, set $v_{n}=P_{C}\left(z_{n}-\lambda_{n} \nabla f\left(z_{n}\right)\right)$. Noticing the firm nonexpansivity of $T_{r_{n}}^{(\Theta, \varphi)}$, we

$$
\begin{aligned}
\| z_{n}- & x^{*} \|^{2} \\
= & \left\|T_{r_{n}}^{(\Theta, \varphi)}\left(x_{n}-r_{n} F x_{n}\right)-T_{r_{n}}^{(\Theta, \varphi)}\left(x^{*}-r_{n} F x^{*}\right)\right\|^{2} \\
\leq & \left\langle\left(x_{n}-r_{n} F x_{n}\right)-\left(x^{*}-r_{n} F x^{*}\right), z_{n}-x^{*}\right\rangle \\
= & \frac{1}{2}\left(\left\|x_{n}-x^{*}-r_{n}\left(F x_{n}-F x^{*}\right)\right\|^{2}+\left\|z_{n}-x^{*}\right\|^{2}-\left\|\left(x_{n}-x^{*}\right)-r_{n}\left(F x_{n}-F x^{*}\right)-\left(z_{n}-x^{*}\right)\right\|^{2}\right) \\
\leq & \frac{1}{2}\left(\left\|x_{n}-x^{*}\right\|^{2}+\left\|z_{n}-x^{*}\right\|^{2}-\left\|\left(x_{n}-z_{n}\right)-r_{n}\left(F x_{n}-F x^{*}\right)\right\|^{2}\right) \\
= & \frac{1}{2}\left(\left\|x_{n}-x^{*}\right\|^{2}+\left\|z_{n}-x^{*}\right\|^{2}-\left\|x_{n}-z_{n}\right\|^{2}+2 r_{n}\left\langle x_{n}-z_{n}, F x_{n}-F x^{*}\right\rangle-r_{n}^{2}\left\|F x_{n}-F x^{*}\right\|^{2}\right), \\
\| v_{n}- & x^{*} \|^{2} \\
= & \left\|P_{C}\left(z_{n}-\lambda_{n} \nabla f\left(z_{n}\right)\right)-P_{C}\left(x^{*}-\lambda_{n} \nabla f\left(x^{*}\right)\right)\right\|^{2} \\
& \leq\left\langle z_{n}-\lambda_{n} \nabla f\left(z_{n}\right)-\left(x^{*}-\lambda_{n} \nabla f\left(x^{*}\right)\right), v_{n}-x^{*}\right\rangle \\
= & \frac{1}{2}\left(\left\|z_{n}-\lambda_{n} \nabla f\left(z_{n}\right)-\left(x^{*}-\lambda_{n} \nabla f\left(x^{*}\right)\right)\right\|^{2}+\left\|v_{n}-x^{*}\right\|^{2}\right. \\
& \left.\quad-\left\|z_{n}-\lambda_{n} \nabla f\left(z_{n}\right)-\left(x^{*}-\lambda_{n} \nabla f\left(x^{*}\right)\right)-\left(v_{n}-x^{*}\right)\right\|^{2}\right) \\
\leq & \frac{1}{2}\left(\left\|z_{n}-x^{*}\right\|^{2}+\left\|v_{n}-x^{*}\right\|^{2}-\left\|z_{n}-v_{n}\right\|^{2}\right. \\
& \left.\quad+2 \lambda_{n}\left\langle\nabla f\left(z_{n}\right)-\nabla f\left(x^{*}\right), z_{n}-v_{n}\right\rangle-\lambda_{n}^{2}\left\|\nabla f\left(z_{n}\right)-\nabla f\left(x^{*}\right)\right\|^{2}\right) \\
\leq & \frac{1}{2}\left(\left\|x_{n}-x^{*}\right\|^{2}+\left\|v_{n}-x^{*}\right\|^{2}-\left\|z_{n}-v_{n}\right\|^{2}+2 \lambda_{n}\left\langle\nabla f\left(z_{n}\right)-\nabla f\left(x^{*}\right), z_{n}-v_{n}\right\rangle\right) .
\end{aligned}
$$

Thus, we have

$$
\begin{gathered}
\left\|z_{n}-x^{*}\right\|^{2} \leq\left\|x_{n}-x^{*}\right\|^{2}-\left\|x_{n}-z_{n}\right\|^{2}+2 r_{n}\left\langle x_{n}-z_{n}, F x_{n}-F x^{*}\right\rangle-r_{n}^{2}\left\|F x_{n}-F x^{*}\right\|^{2}, \\
\left\|v_{n}-x^{*}\right\|^{2} \leq\left\|x_{n}-x^{*}\right\|^{2}-\left\|z_{n}-v_{n}\right\|^{2}+2 \lambda_{n}\left\|\nabla f\left(z_{n}\right)-\nabla f\left(x^{*}\right)\right\|\left\|z_{n}-v_{n}\right\| .
\end{gathered}
$$

It follows that

$$
\begin{aligned}
\left\|y_{n}-x^{*}\right\|^{2} & \leq \alpha_{n}\left\|Q x_{n}-x^{*}\right\|^{2}+\left(1-\alpha_{n}\right)\left\|v_{n}-x^{*}\right\|^{2} \\
& \leq \alpha_{n}\left\|Q x_{n}-x^{*}\right\|^{2}+\left\|v_{n}-x^{*}\right\|^{2} \\
& \leq \alpha_{n}\left\|Q x_{n}-x^{*}\right\|^{2}+\left\|x_{n}-x^{*}\right\|^{2}-\left\|z_{n}-v_{n}\right\|^{2}+2 \lambda_{n}\left\|\nabla f\left(z_{n}\right)-\nabla f\left(x^{*}\right)\right\|\left\|z_{n}-v_{n}\right\| .
\end{aligned}
$$


From (3.18), (3.19), and (3.25), we have

$$
\begin{aligned}
\left\|x_{n+1}-x^{*}\right\|^{2} \leq & \beta_{n}\left\|x_{n}-x^{*}\right\|^{2}+\left(1-\beta_{n}\right) \alpha_{n}\left\|Q x_{n}-x^{*}\right\|^{2}+\left(1-\beta_{n}\right)\left\|z_{n}-x^{*}\right\|^{2}+M \alpha_{n} \\
\leq & \left\|x_{n}-x^{*}\right\|^{2}-\left(1-\beta_{n}\right)\left\|x_{n}-z_{n}\right\|^{2}+2\left(1-\beta_{n}\right) r_{n}\left\|x_{n}-z_{n}\right\|\left\|F x_{n}-F x^{*}\right\| \\
& +\left(M+\left\|Q x_{n}-x^{*}\right\|^{2}\right) \alpha_{n} .
\end{aligned}
$$

It follows that

$$
\begin{aligned}
\left(1-\beta_{n}\right)\left\|x_{n}-z_{n}\right\|^{2} \leq & \left(\left\|x_{n}-x^{*}\right\|+\left\|x_{n+1}-x^{*}\right\|\right)\left\|x_{n+1}-x_{n}\right\|+\left(M+\left\|Q x_{n}-x^{*}\right\|^{2}\right) \alpha_{n} \\
& +2\left(1-\beta_{n}\right) r_{n}\left\|x_{n}-z_{n}\right\|\left\|F x_{n}-F x^{*}\right\| .
\end{aligned}
$$

Note that $\left\|x_{n+1}-x_{n}\right\| \rightarrow 0, \alpha_{n} \rightarrow 0$ and $\left\|F x_{n}-F x^{*}\right\| \rightarrow 0$. Then we immediately deduce that

$$
\lim _{n \rightarrow \infty}\left\|x_{n}-z_{n}\right\|=0
$$

From (3.19) and (3.27), we have

$$
\begin{aligned}
\left\|x_{n+1}-x^{*}\right\|^{2} \leq & \left\|x_{n}-x^{*}\right\|^{2}-\left(1-\beta_{n}\right)\left\|z_{n}-v_{n}\right\|^{2} \\
& +2 \lambda_{n}\left(1-\beta_{n}\right)\left\|\nabla f\left(z_{n}\right)-\nabla f\left(x^{*}\right)\right\|\left\|z_{n}-v_{n}\right\|+\left(M+\left\|Q x_{n}-x^{*}\right\|^{2}\right) \alpha_{n} .
\end{aligned}
$$

So, we obtain

$$
\begin{aligned}
\left(1-\beta_{n}\right)\left\|z_{n}-v_{n}\right\|^{2} \leq & \left(\left\|x_{n}-x^{*}\right\|+\left\|x_{n+1}-x^{*}\right\|\right)\left\|x_{n+1}-x_{n}\right\| \\
& +\left(M+\left\|Q x_{n}-x^{*}\right\|^{2}\right) \alpha_{n}+2 \lambda_{n}\left(1-\beta_{n}\right)\left\|\nabla f\left(z_{n}\right)-\nabla f\left(x^{*}\right)\right\|\left\|z_{n}-v_{n}\right\| .
\end{aligned}
$$

Note that $\left\|x_{n+1}-x_{n}\right\| \rightarrow 0, \alpha_{n} \rightarrow 0$ and $\left\|\nabla f\left(z_{n}\right)-\nabla f\left(x^{*}\right)\right\| \rightarrow 0$. Then we immediately conclude that

$$
\lim _{n \rightarrow \infty}\left\|z_{n}-v_{n}\right\|=0
$$

This together with $\left\|y_{n}-v_{n}\right\| \leq \alpha_{n}\left\|Q x_{n}-v_{n}\right\| \rightarrow 0$, implies that

$$
\lim _{n \rightarrow \infty}\left\|z_{n}-y_{n}\right\|=0
$$

Thus, from (3.30) and (3.34), we deduce that

$$
\lim _{n \rightarrow \infty}\left\|x_{n}-y_{n}\right\|=0 .
$$


Since

$$
\begin{aligned}
\left\|\delta_{n}\left(S y_{n}-x_{n}\right)\right\| & \leq\left\|x_{n+1}-x_{n}\right\|+\gamma_{n}\left\|P_{C}\left(z_{n}-\lambda_{n} \nabla f\left(z_{n}\right)\right)-x_{n}\right\| \\
& \leq\left\|x_{n+1}-x_{n}\right\|+\gamma_{n}\left\|y_{n}-x_{n}\right\|+\gamma_{n} \alpha_{n}\left\|Q x_{n}-P_{C}\left(z_{n}-\lambda_{n} \nabla f\left(z_{n}\right)\right)\right\| .
\end{aligned}
$$

Therefore,

$$
\lim _{n \rightarrow \infty}\left\|S y_{n}-x_{n}\right\|=0, \quad \lim _{n \rightarrow \infty}\left\|S y_{n}-y_{n}\right\|=0
$$

Step 4. We claim that $\lim \sup _{n \rightarrow \infty}\left\langle Q x^{*}-x^{*}, x_{n}-x^{*}\right\rangle \leq 0$ where $x^{*}=P_{\mathrm{Fix}(S) \cap \Omega \cap G M E P} Q x^{*}$. $u$ and

Indeed, since $\left\{x_{n}\right\}$ is bounded, there exists a subsequence $\left\{x_{n_{i}}\right\}$ of $\left\{x_{n}\right\}$ such that $x_{n_{i}} \rightarrow$

$$
\limsup _{n \rightarrow \infty}\left\langle Q x^{*}-x^{*}, x_{n}-x^{*}\right\rangle=\lim _{i \rightarrow \infty}\left\langle Q x^{*}-x^{*}, x_{n_{i}}-x^{*}\right\rangle=\left\langle Q x^{*}-x^{*}, u-x^{*}\right\rangle .
$$

We can obtain that $u \in \operatorname{Fix}(S) \cap \Omega \cap$ GMEP. First, we show $u \in \Omega(=\operatorname{VI}(C, \nabla f))$. Since $x_{n}-z_{n} \rightarrow 0$ and $v_{n}-z_{n} \rightarrow 0$, we conclude that $z_{n_{i}} \rightarrow u$ and $v_{n_{i}} \rightarrow u$. Let

$$
T v= \begin{cases}\nabla f(v)+N_{C} v, & \text { if } v \in C, \\ \emptyset, & \text { if } v \notin C,\end{cases}
$$

where $N_{C} v$ is the normal cone to $C$ at $v \in C$. We have already mentioned that in this case, the mapping $T$ is maximal monotone, and $0 \in T v$ if and only if $v \in \operatorname{VI}(C, \nabla f)(=\Omega)$; see [37]. Let $G(T)$ be the graph of $T$ and let $(v, w) \in G(T)$. Then, we have $w \in T v=\nabla f(v)+N_{C} v$ and hence $w-\nabla f(v) \in N_{C} v$. So, we have $\langle v-t, w-\nabla f(v)\rangle \geq 0$ for all $t \in C$. On the other hand, from $v_{n}=P_{C}\left(z_{n}-\lambda_{n} \nabla f\left(z_{n}\right)\right)$ and $v \in C$, we have

$$
\left\langle z_{n}-\lambda_{n} \nabla f\left(z_{n}\right)-v_{n}, v_{n}-v\right\rangle \geq 0
$$

and hence

$$
\left\langle v-v_{n}, \frac{v_{n}-z_{n}}{\lambda_{n}}+\nabla f\left(z_{n}\right)\right\rangle \geq 0
$$

From $\langle v-t, w-\nabla f(v)\rangle \geq 0$ for all $t \in C$ and $v_{n_{i}} \in C$, we have

$$
\begin{aligned}
\langle v- & \left.v_{n_{i}}, w\right\rangle \\
& \geq\left\langle v-v_{n_{i}}, \nabla f(v)\right\rangle \\
& \geq\left\langle v-v_{n_{i}}, \nabla f(v)\right\rangle-\left\langle v-v_{n_{i}}, \frac{v_{n_{i}}-z_{n_{i}}}{\lambda_{n_{i}}}+\nabla f\left(z_{n_{i}}\right)\right\rangle \\
& =\left\langle v-v_{n_{i}}, \nabla f(v)-\nabla f\left(v_{n_{i}}\right)\right\rangle+\left\langle v-v_{n_{i}}, \nabla f\left(v_{n_{i}}\right)-\nabla f\left(z_{n_{i}}\right)\right\rangle-\left\langle v-v_{n_{i}}, \frac{v_{n_{i}}-z_{n_{i}}}{\lambda_{n_{i}}}\right\rangle \\
& \geq\left\langle v-v_{n_{i}}, \nabla f\left(v_{n_{i}}\right)-\nabla f\left(z_{n_{i}}\right)\right\rangle-\left\langle v-v_{n_{i}}, \frac{v_{n_{i}}-z_{n_{i}}}{\lambda_{n_{i}}}\right\rangle .
\end{aligned}
$$


Hence, we obtain $\langle v-u, w\rangle \geq 0$ as $i \rightarrow \infty$. Since $T$ is maximal monotone, we have $u \in T^{-1} 0$ and hence $u \in \operatorname{VI}(C, \nabla f)(=\Omega)$.

Secondly, let us show $u \in \operatorname{Fix}(S)$. Since $x_{n}-y_{n} \rightarrow 0$ and $x_{n_{i}} \rightarrow u$, we have $y_{n_{i}} \rightarrow u$. Also, since $y_{n}-S y_{n} \rightarrow 0$, it follows that $y_{n_{i}}-S y_{n_{i}} \rightarrow 0$ as $i \rightarrow \infty$. So, in terms of Proposition 2.5(ii) we obtain $u \in \operatorname{Fix}(S)$.

Next, let us show $u \in$ GMEP. From $z_{n}=T_{r_{n}}^{(\Theta, \varphi)}\left(x_{n}-r_{n} F x_{n}\right)$, we know that

$$
\Theta\left(z_{n}, y\right)+\varphi(y)-\varphi\left(z_{n}\right)+\left\langle F x_{n}, y-z_{n}\right\rangle+\frac{1}{r_{n}}\left\langle y-z_{n}, z_{n}-x_{n}\right\rangle \geq 0, \quad \forall y \in C .
$$

From (H2), it follows that

$$
\varphi(y)-\varphi\left(z_{n}\right)+\left\langle F x_{n}, y-z_{n}\right\rangle+\frac{1}{r_{n}}\left\langle y-z_{n}, z_{n}-x_{n}\right\rangle \geq \Theta\left(y, z_{n}\right), \quad \forall y \in C .
$$

Replacing $n$ by $n_{i}$, we have

$$
\varphi(y)-\varphi\left(z_{n_{i}}\right)+\left\langle F x_{n_{i}}, y-z_{n_{i}}\right\rangle+\left\langle y-z_{n_{i}}, \frac{z_{n_{i}}-x_{n_{i}}}{r_{n_{i}}}\right\rangle \geq \Theta\left(y, z_{n_{i}}\right), \quad \forall y \in C .
$$

Put $z_{s}=s y+(1-s) u$ for all $s \in(0,1]$ and $y \in C$. Then, we have $z_{s} \in C$. So, from (3.45), we have

$$
\begin{aligned}
\left\langle z_{s}-z_{n_{i}}, F z_{s}\right\rangle \geq & \left\langle z_{s}-z_{n_{i}}, F z_{s}\right\rangle-\varphi\left(z_{s}\right)+\varphi\left(z_{n_{i}}\right)-\left\langle z_{s}-z_{n_{i}}, F x_{n_{i}}\right\rangle \\
& -\left\langle z_{s}-z_{n_{i}}, \frac{z_{n_{i}}-x_{n_{i}}}{r_{n_{i}}}\right\rangle+\Theta\left(z_{s}, z_{n_{i}}\right) \\
= & \left\langle z_{s}-z_{n_{i}}, F z_{s}-F z_{n_{i}}\right\rangle+\left\langle z_{s}-z_{n_{i}}, F z_{n_{i}}-F x_{n_{i}}\right\rangle-\varphi\left(z_{s}\right)+\varphi\left(z_{n_{i}}\right) \\
& -\left\langle z_{s}-z_{n_{i}}, \frac{z_{n_{i}}-x_{n_{i}}}{r_{n_{i}}}\right\rangle+\Theta\left(z_{s}, z_{n_{i}}\right) .
\end{aligned}
$$

Since $\left\|z_{n_{i}}-x_{n_{i}}\right\| \rightarrow 0$, we have $\left\|F z_{n_{i}}-F x_{n_{i}}\right\| \rightarrow 0$. Further, from the monotonicity of $F$, we have $\left\langle z_{s}-z_{n_{i}}, F z_{s}-F z_{n_{i}}\right\rangle \geq 0$. So, from (H4), the weakly lower semicontinuity of $\varphi,\left(z_{n_{i}}-x_{n_{i}}\right) / r_{n_{i}} \rightarrow$ 0 and $z_{n_{i}} \rightarrow u$, we have

$$
\left\langle z_{s}-z_{n_{i}}, F z_{s}\right\rangle \geq-\varphi\left(z_{s}\right)+\varphi(u)+\Theta\left(z_{s}, u\right)
$$

as $i \rightarrow \infty$. From (H1), (H4), and (3.47), we also have

$$
\begin{aligned}
0 & =\Theta\left(z_{s}, z_{s}\right)+\varphi\left(z_{s}\right)+\varphi\left(z_{s}\right) \\
& \leq s \Theta\left(z_{s}, y\right)+(1-s) \Theta\left(z_{s}, u\right)+s \varphi(y)+(1-s) \varphi(u)-\varphi\left(z_{s}\right) \\
& =s\left[\Theta\left(z_{s}, y\right)+\varphi(y)-\varphi\left(z_{s}\right)\right]+(1-s)\left[\Theta\left(z_{s}, u\right)+\varphi(u)-\varphi\left(z_{s}\right)\right] \\
& \leq s\left[\Theta\left(z_{s}, y\right)+\varphi(y)-\varphi\left(z_{s}\right)\right]+(1-s)\left\langle z_{s}-u, F z_{s}\right\rangle \\
& =s\left[\Theta\left(z_{s}, y\right)+\varphi(y)-\varphi\left(z_{s}\right)\right]+(1-s) s\left\langle y-u, F z_{s}\right\rangle,
\end{aligned}
$$


and hence

$$
0 \leq \Theta\left(z_{s}, y\right)+\varphi(y)-\varphi\left(z_{s}\right)+(1-s)\left\langle y-u, F z_{s}\right\rangle
$$

Letting $s \rightarrow 0$, we have, for each $y \in C$,

$$
0 \leq \Theta(u, y)+\varphi(y)-\varphi(u)+\langle y-u, F u\rangle
$$

This shows that $u \in$ GMEP. Therefore, $u \in \operatorname{Fix}(S) \cap \Omega \cap$ GMEP. Hence, it follows from (2.4) that

$$
\limsup _{n \rightarrow \infty}\left\langle Q x^{*}-x^{*}, x_{n}-x^{*}\right\rangle=\lim _{i \rightarrow \infty}\left\langle Q x^{*}-x^{*}, x_{n_{i}}-x^{*}\right\rangle=\left\langle Q x^{*}-x^{*}, u-x^{*}\right\rangle \leq 0 .
$$

Step 5. We claim that $\lim _{n \rightarrow \infty}\left\|x_{n}-x^{*}\right\|=0$.

Indeed, from (3.2) and the convexity of $\|\cdot\|$, we have

$$
\begin{aligned}
\| x_{n+1}- & x^{*} \|^{2} \\
= & \left\|\beta_{n}\left(x_{n}-x^{*}\right)+\gamma_{n}\left(y_{n}-x^{*}\right)+\delta_{n}\left(S y_{n}-x^{*}\right)+\gamma_{n} \alpha_{n}\left(P_{C}\left(z_{n}-\lambda_{n} \nabla f\left(z_{n}\right)\right)-Q x_{n}\right)\right\|^{2} \\
\leq & \left\|\beta_{n}\left(x_{n}-x^{*}\right)+\gamma_{n}\left(y_{n}-x^{*}\right)+\delta_{n}\left(S y_{n}-x^{*}\right)\right\|^{2} \\
& +2 \gamma_{n} \alpha_{n}\left\langle P_{C}\left(z_{n}-\lambda_{n} \nabla f\left(z_{n}\right)\right)-Q x_{n}, x_{n+1}-x^{*}\right\rangle \\
\leq & \beta_{n}\left\|x_{n}-x^{*}\right\|^{2}+\left(1-\beta_{n}\right)\left\|\frac{1}{1-\beta_{n}}\left[\gamma_{n}\left(y_{n}-x^{*}\right)+\delta_{n}\left(S y_{n}-x^{*}\right)\right]\right\|^{2} \\
& +2 \gamma_{n} \alpha_{n}\left\langle P_{C}\left(z_{n}-\lambda_{n} \nabla f\left(z_{n}\right)\right)-x^{*}, x_{n+1}-x^{*}\right\rangle+2 \gamma_{n} \alpha_{n}\left\langle x^{*}-Q x_{n}, x_{n+1}-x^{*}\right\rangle .
\end{aligned}
$$

Utilizing Lemma 3.1, we get from (3.52)

$$
\begin{aligned}
\| x_{n+1}- & x^{*} \|^{2} \\
\leq & \beta_{n}\left\|x_{n}-x^{*}\right\|^{2}+\left(1-\beta_{n}\right)\left\|y_{n}-x^{*}\right\|^{2}+2 \gamma_{n} \alpha_{n}\left\|P_{C}\left(z_{n}-\lambda_{n} \nabla f\left(z_{n}\right)\right)-x^{*}\right\|\left\|x_{n+1}-x^{*}\right\| \\
& +2 \gamma_{n} \alpha_{n}\left\langle x^{*}-Q x_{n}, x_{n+1}-x^{*}\right\rangle \\
\leq & \beta_{n}\left\|x_{n}-x^{*}\right\|^{2}+\left(1-\beta_{n}\right)\left[\left(1-\alpha_{n}\right)\left\|z_{n}-x^{*}\right\|^{2}+2 \alpha_{n}\left\langle Q x_{n}-x^{*}, y_{n}-x^{*}\right\rangle\right] \\
& +2 \gamma_{n} \alpha_{n}\left\|z_{n}-x^{*}\right\|\left\|x_{n+1}-x^{*}\right\|+2 \gamma_{n} \alpha_{n}\left\langle x^{*}-Q x_{n}, x_{n+1}-x^{*}\right\rangle .
\end{aligned}
$$


From (3.17), we note that $\left\|z_{n}-x^{*}\right\| \leq\left\|x_{n}-x^{*}\right\|$. Hence we have

$$
\begin{aligned}
\| x_{n+1}- & x^{*} \|^{2} \\
\leq & \beta_{n}\left\|x_{n}-x^{*}\right\|^{2}+\left(1-\beta_{n}\right)\left(1-\alpha_{n}\right)\left\|x_{n}-x^{*}\right\|^{2}+2 \alpha_{n}\left(1-\beta_{n}\right)\left\langle Q x_{n}-x^{*}, y_{n}-x^{*}\right\rangle \\
& +2 \gamma_{n} \alpha_{n}\left\|x_{n}-x^{*}\right\|\left\|x_{n+1}-x^{*}\right\|+2 \gamma_{n} \alpha_{n}\left\langle x^{*}-Q x_{n}, x_{n+1}-x^{*}\right\rangle \\
\leq & {\left[1-\left(1-\beta_{n}\right) \alpha_{n}\right]\left\|x_{n}-x^{*}\right\|^{2}+2 \alpha_{n} \gamma_{n}\left\langle Q x_{n}-x^{*}, y_{n}-x_{n+1}\right\rangle } \\
& +2 \alpha_{n} \delta_{n}\left\langle Q x_{n}-x^{*}, y_{n}-x^{*}\right\rangle+2 \alpha_{n} \gamma_{n}\left\|x_{n}-x^{*}\right\|\left\|x_{n+1}-x^{*}\right\| \\
\leq & {\left[1-\left(1-\beta_{n}\right) \alpha_{n}\right]\left\|x_{n}-x^{*}\right\|^{2}+2 \alpha_{n} \gamma_{n}\left\|Q x_{n}-x^{*}\right\|\left\|y_{n}-x_{n+1}\right\| } \\
& +2 \alpha_{n} \delta_{n}\left\langle Q x_{n}-x^{*}, x_{n}-x^{*}\right\rangle+2 \alpha_{n} \delta_{n}\left\langle Q x_{n}-x^{*}, y_{n}-x_{n}\right\rangle+2 \alpha_{n} \gamma_{n}\left\|x_{n}-x^{*}\right\|\left\|x_{n+1}-x^{*}\right\| \\
\leq & {\left[1-\left(1-\beta_{n}\right) \alpha_{n}\right]\left\|x_{n}-x^{*}\right\|^{2}+2 \alpha_{n} \gamma_{n}\left\|Q x_{n}-x^{*}\right\|\left\|y_{n}-x_{n+1}\right\| } \\
& +2 \alpha_{n} \delta_{n} \rho\left\|x_{n}-x^{*}\right\|^{2}+2 \alpha_{n} \delta_{n}\left\langle Q x^{*}-x^{*}, x_{n}-x^{*}\right\rangle \\
& +2 \alpha_{n} \delta_{n}\left\|Q x_{n}-x^{*}\right\|\left\|y_{n}-x_{n}\right\|+2 \alpha_{n} \gamma_{n}\left\|x_{n}-x^{*}\right\|\left\|x_{n+1}-x^{*}\right\| \\
\leq & {\left[1-\left(1-\beta_{n}\right) \alpha_{n}\right]\left\|x_{n}-x^{*}\right\|^{2}+2 \alpha_{n} \gamma_{n}\left\|Q x_{n}-x^{*}\right\|\left\|y_{n}-x_{n+1}\right\| } \\
& +2 \alpha_{n} \delta_{n} \rho\left\|x_{n}-x^{*}\right\|^{2}+2 \alpha_{n} \delta_{n}\left\langle Q x^{*}-x^{*}, x_{n}-x^{*}\right\rangle \\
& +2 \alpha_{n} \delta_{n}\left\|Q x_{n}-x^{*}\right\|\left\|y_{n}-x_{n}\right\|+\alpha_{n} \gamma_{n}\left(\left\|x_{n}-x^{*}\right\|^{2}+\left\|x_{n+1}-x^{*}\right\|^{2}\right),
\end{aligned}
$$

that is,

$$
\begin{aligned}
\| x_{n+1}- & x^{*} \|^{2} \\
\leq & {\left[1-\frac{(1-2 \rho) \delta_{n}-\gamma_{n}}{1-\alpha_{n} \gamma_{n}} \alpha_{n}\right]\left\|x_{n}-x^{*}\right\|^{2}+\frac{\left[(1-2 \rho) \delta_{n}-\gamma_{n}\right] \alpha_{n}}{1-\alpha_{n} \gamma_{n}} } \\
& \times\left\{\frac{2 \gamma_{n}}{(1-2 \rho) \delta_{n}-\gamma_{n}}\left\|Q x_{n}-x^{*}\right\|\left\|y_{n}-x_{n+1}\right\|+\frac{2 \delta_{n}}{(1-2 \rho) \delta_{n}-\gamma_{n}}\left\|Q x_{n}-x^{*}\right\|\left\|y_{n}-x_{n}\right\|\right. \\
& \left.+\frac{2 \delta_{n}}{(1-2 \rho) \delta_{n}-\gamma_{n}}\left\langle Q x^{*}-x^{*}, x_{n}-x^{*}\right\rangle\right\} .
\end{aligned}
$$

Note that $\liminf _{n \rightarrow \infty}\left((1-2 \rho) \delta_{n}-\gamma_{n}\right) /\left(1-\alpha_{n} \gamma_{n}\right)>0$. It follows that $\left(\sum_{n=0}^{\infty}\left((1-2 \rho) \delta_{n}-\gamma_{n}\right) /(1-\right.$ $\left.\left.\alpha_{n} \gamma_{n}\right)\right) \alpha_{n}=\infty$. It is clear that

$$
\begin{aligned}
\limsup _{n \rightarrow \infty}\left\{\frac{2 \gamma_{n}}{(1-2 \rho) \delta_{n}-\gamma_{n}}\left\|Q x_{n}-x^{*}\right\|\left\|y_{n}-x_{n+1}\right\|+\frac{2 \delta_{n}}{(1-2 \rho) \delta_{n}-\gamma_{n}}\left\|Q x_{n}-x^{*}\right\|\left\|y_{n}-x_{n}\right\|\right. \\
\left.\quad+\frac{2 \delta_{n}}{(1-2 \rho) \delta_{n}-\gamma_{n}}\left\langle Q x^{*}-x^{*}, x_{n}-x^{*}\right\rangle\right\} \leq 0 .
\end{aligned}
$$


Therefore, all conditions of Lemma 2.7 are satisfied. This immediately implies that $x_{n} \rightarrow x^{*}$. It is readily seen that both $\left\{y_{n}\right\}$ and $\left\{z_{n}\right\}$ converge strongly to the same point $x^{*}$. The proof is complete.

Utilizing Theorem 3.2, we establish the following corollaries.

Corollary 3.3. Let $C$ be a nonempty bounded closed convex subset of a real Hilbert space H. Let $\Theta: C \times C \rightarrow \mathbf{R}$ be a bifunction satisfying conditions (H1)-(H4) and $\varphi: C \rightarrow \mathbf{R}$ be a lower semicontinuous and convex function with assumptions (A1) or (A2). Suppose the minimization (1.5) is consistent and let $\Omega$ denote its solution set. Assume the gradient $\nabla f$ is L-Lipschitzian with constant $L>0$ and $F: C \rightarrow H$ is an $\alpha$-inverse strongly monotone mapping. Let $S: C \rightarrow C$ be a $k$-strictly pseudocontractive mapping such that $\operatorname{Fix}(S) \cap \Omega \cap \mathrm{GMEP} \neq \emptyset$. For fixed $u \in C$ and given $x_{0} \in C$ arbitrarily, let the sequences $\left\{x_{n}\right\},\left\{y_{n}\right\}$ and $\left\{z_{n}\right\}$ be generated iteratively by

$$
\begin{gathered}
\Theta\left(z_{n}, y\right)+\varphi(y)-\varphi\left(z_{n}\right)+\left\langle F x_{n}, y-z_{n}\right\rangle+\frac{1}{r_{n}}\left\langle y-z_{n}, z_{n}-x_{n}\right\rangle \geq 0, \quad \forall y \in C, \\
y_{n}=\alpha_{n} u+\left(1-\alpha_{n}\right) P_{C}\left(z_{n}-\lambda_{n} \nabla f\left(z_{n}\right)\right), \\
x_{n+1}=\beta_{n} x_{n}+\gamma_{n} P_{C}\left(z_{n}-\lambda_{n} \nabla f\left(z_{n}\right)\right)+\delta_{n} S y_{n}, \quad \forall n \geq 0,
\end{gathered}
$$

where $\left\{\lambda_{n}\right\} \subset(0,2 / L],\left\{r_{n}\right\} \subset(0,2 \alpha]$, and $\left\{\alpha_{n}\right\},\left\{\beta_{n}\right\},\left\{\gamma_{n}\right\},\left\{\delta_{n}\right\}$ are four sequences in $[0,1]$ such that:

(i) $0<\liminf _{n \rightarrow \infty} \lambda_{n} \leq \lim \sup _{n \rightarrow \infty} \lambda_{n}<2 / L$ and $\lim _{n \rightarrow \infty}\left(\lambda_{n}-\lambda_{n+1}\right)=0$;

(ii) $0<\liminf _{n \rightarrow \infty} r_{n} \leq \limsup _{n \rightarrow \infty} r_{n}<2 \alpha$ and $\lim _{n \rightarrow \infty}\left(r_{n}-r_{n+1}\right)=0$;

(iii) $\beta_{n}+\gamma_{n}+\delta_{n}=1$ and $\left(\gamma_{n}+\delta_{n}\right) k \leq \gamma_{n}<\delta_{n}$ for all $n \geq 0$;

(iv) $\lim _{n \rightarrow \infty} \alpha_{n}=0$ and $\sum_{n=0}^{\infty} \alpha_{n}=\infty$;

(v) $0<\liminf _{n \rightarrow \infty} \beta_{n} \leq \lim \sup _{n \rightarrow \infty} \beta_{n}<1$ and $\liminf _{n \rightarrow \infty} \delta_{n}>0$;

(vi) $\lim _{n \rightarrow \infty}\left(\gamma_{n+1} /\left(1-\beta_{n+1}\right)-\gamma_{n} /\left(1-\beta_{n}\right)\right)=0$.

Then, $\left\{x_{n}\right\},\left\{y_{n}\right\}$ and $\left\{z_{n}\right\}$ converge strongly to the same point $x^{*}=P_{\mathrm{Fix}(S) \cap \Omega \cap G M E P} u$.

Corollary 3.4. Let $C$ be a nonempty bounded closed convex subset of a real Hilbert space H. Let $\Theta: C \times C \rightarrow \mathbf{R}$ be a bifunction satisfying conditions (H1)-(H4) and $\varphi: C \rightarrow \mathbf{R}$ be a lower semicontinuous and convex function with assumptions (A1) or (A2). Suppose the minimization (1.5) is consistent and let $\Omega$ denote its solution set. Assume the gradient $\nabla f$ is L-Lipschitzian with constant $L>0$ and $F: C \rightarrow H$ is an $\alpha$-inverse strongly monotone mapping. Let $S: C \rightarrow C$ be a nonexpansive mapping such that $\operatorname{Fix}(S) \cap \Omega \cap \mathrm{GMEP} \neq \emptyset$. Let $Q: C \rightarrow C$ be a $\rho$-contraction with $\rho \in[0,1 / 2)$. For given $x_{0} \in C$ arbitrarily, let the sequences $\left\{x_{n}\right\},\left\{y_{n}\right\}$ and $\left\{z_{n}\right\}$ be generated iteratively by

$$
\begin{gathered}
\Theta\left(z_{n}, y\right)+\varphi(y)-\varphi\left(z_{n}\right)+\left\langle F x_{n}, y-z_{n}\right\rangle+\frac{1}{r_{n}}\left\langle y-z_{n}, z_{n}-x_{n}\right\rangle \geq 0, \quad \forall y \in C, \\
y_{n}=\alpha_{n} Q x_{n}+\left(1-\alpha_{n}\right) P_{C}\left(z_{n}-\lambda_{n} \nabla f\left(z_{n}\right)\right), \\
x_{n+1}=\beta_{n} x_{n}+\gamma_{n} P_{C}\left(z_{n}-\lambda_{n} \nabla f\left(z_{n}\right)\right)+\delta_{n} S y_{n}, \quad \forall n \geq 0,
\end{gathered}
$$

where $\left\{\lambda_{n}\right\} \subset(0,2 / L],\left\{r_{n}\right\} \subset(0,2 \alpha]$ and $\left\{\alpha_{n}\right\},\left\{\beta_{n}\right\},\left\{\gamma_{n}\right\},\left\{\delta_{n}\right\}$ are four sequences in $[0,1]$ such that 
(i) $0<\liminf _{n \rightarrow \infty} \lambda_{n} \leq \limsup \sup _{n \rightarrow \infty} \lambda_{n}<2 / L$ and $\lim _{n \rightarrow \infty}\left(\lambda_{n}-\lambda_{n+1}\right)=0$;

(ii) $0<\liminf _{n \rightarrow \infty} r_{n} \leq \limsup _{n \rightarrow \infty} r_{n}<2 \alpha$ and $\lim _{n \rightarrow \infty}\left(r_{n}-r_{n+1}\right)=0$;

(iii) $\beta_{n}+\gamma_{n}+\delta_{n}=1$ and $\gamma_{n}<(1-2 \rho) \delta_{n}$ for all $n \geq 0$;

(iv) $\lim _{n \rightarrow \infty} \alpha_{n}=0$ and $\sum_{n=0}^{\infty} \alpha_{n}=\infty$;

(v) $0<\liminf _{n \rightarrow \infty} \beta_{n} \leq \lim \sup _{n \rightarrow \infty} \beta_{n}<1$ and $\liminf _{n \rightarrow \infty} \gamma_{n}>0$;

(vi) $\lim _{n \rightarrow \infty}\left(\gamma_{n+1} /\left(1-\beta_{n+1}\right)-\gamma_{n} /\left(1-\beta_{n}\right)\right)=0$.

Then $\left\{x_{n}\right\},\left\{y_{n}\right\}$, and $\left\{z_{n}\right\}$ converge strongly to the same point $x^{*}=P_{\mathrm{Fix}(S) \cap \Omega \cap G \mathrm{MEP}} Q x^{*}$.

Corollary 3.5. Let $C$ be a nonempty bounded closed convex subset of a real Hilbert space $H$. Let $\Theta$ : $\mathrm{C} \times \mathrm{C} \rightarrow \mathbf{R}$ be a bifunction satisfying conditions (H1)-(H4) and $\varphi: C \rightarrow \mathbf{R}$ a lower semicontinuous and convex function with assumptions (A1) or (A2). Suppose the minimization (1.5) is consistent and let $\Omega$ denote its solution set. Assume the gradient $\nabla f$ is L-Lipschitzian with constant $L>0$ and $F: C \rightarrow H$ is an $\alpha$-inverse strongly monotone mapping. Let $S: C \rightarrow C$ be a nonexpansive mapping such that $\operatorname{Fix}(S) \cap \Omega \cap \mathrm{GMEP} \neq \emptyset$. For fixed $u \in C$ and given $x_{0} \in C$ arbitrarily, let the sequences $\left\{x_{n}\right\},\left\{y_{n}\right\}$, and $\left\{z_{n}\right\}$ be generated iteratively by

$$
\begin{gathered}
\Theta\left(z_{n}, y\right)+\varphi(y)-\varphi\left(z_{n}\right)+\left\langle F x_{n}, y-z_{n}\right\rangle+\frac{1}{r_{n}}\left\langle y-z_{n}, z_{n}-x_{n}\right\rangle \geq 0, \quad \forall y \in C, \\
y_{n}=\alpha_{n} u+\left(1-\alpha_{n}\right) P_{C}\left(z_{n}-\lambda_{n} \nabla f\left(z_{n}\right)\right), \\
x_{n+1}=\beta_{n} x_{n}+\gamma_{n} P_{C}\left(z_{n}-\lambda_{n} \nabla f\left(z_{n}\right)\right)+\delta \delta_{n} S y_{n}, \quad \forall n \geq 0,
\end{gathered}
$$

where $\left\{\lambda_{n}\right\} \subset(0,2 / L],\left\{r_{n}\right\} \subset(0,2 \alpha]$, and $\left\{\alpha_{n}\right\},\left\{\beta_{n}\right\},\left\{\gamma_{n}\right\},\left\{\delta_{n}\right\}$ are four sequences in $[0,1]$ such that:

(i) $0<\liminf _{n \rightarrow \infty} \lambda_{n} \leq \limsup \sup _{n \rightarrow \infty} \lambda_{n}<2 / L$ and $\lim _{n \rightarrow \infty}\left(\lambda_{n}-\lambda_{n+1}\right)=0$;

(ii) $0<\liminf _{n \rightarrow \infty} r_{n} \leq \limsup _{n \rightarrow \infty} r_{n}<2 \alpha$ and $\lim _{n \rightarrow \infty}\left(r_{n}-r_{n+1}\right)=0$;

(iii) $\beta_{n}+\gamma_{n}+\delta_{n}=1$ and $\gamma_{n}<\delta_{n}$ for all $n \geq 0$;

(iv) $\lim _{n \rightarrow \infty} \alpha_{n}=0$ and $\sum_{n=0}^{\infty} \alpha_{n}=\infty$;

(v) $0<\liminf _{n \rightarrow \infty} \beta_{n} \leq \lim \sup _{n \rightarrow \infty} \beta_{n}<1$ and $\liminf _{n \rightarrow \infty} \gamma_{n}>0$;

(vi) $\lim _{n \rightarrow \infty}\left(\gamma_{n+1} /\left(1-\beta_{n+1}\right)-\gamma_{n} /\left(1-\beta_{n}\right)\right)=0$.

Then, $\left\{x_{n}\right\},\left\{y_{n}\right\}$, and $\left\{z_{n}\right\}$ converge strongly to the same point $x^{*}=P_{\operatorname{Fix}(S) \cap \Omega \cap G M E P} u$.

Corollary 3.6. Let $C$ be a nonempty bounded closed convex subset of a real Hilbert space H. Suppose the minimization (1.5) is consistent and let $\Omega$ denote its solution set. Assume the gradient $\nabla f$ is $L$ Lipschitzian with constant $L>0$ and $A: C \rightarrow H$ is an $\alpha$-inverse strongly monotone mapping. Let $S: C \rightarrow C$ be a k-strictly pseudocontractive mapping such that $\operatorname{Fix}(S) \cap \Omega \cap \operatorname{VI}(C, A) \neq \emptyset$. For fixed $u \in C$ and given $x_{0} \in C$ arbitrarily, let the sequences $\left\{x_{n}\right\},\left\{y_{n}\right\}$, and $\left\{z_{n}\right\}$ be generated iteratively by

$$
\begin{gathered}
z_{n}=P_{C}\left(x_{n}-r_{n} A x_{n}\right), \\
y_{n}=\alpha_{n} u+\left(1-\alpha_{n}\right) P_{C}\left(z_{n}-\lambda_{n} \nabla f\left(z_{n}\right)\right), \\
x_{n+1}=\beta_{n} x_{n}+\gamma_{n} P_{C}\left(z_{n}-\lambda_{n} \nabla f\left(z_{n}\right)\right)+\delta_{n} S y_{n}, \quad \forall n \geq 0,
\end{gathered}
$$


where $\left\{\lambda_{n}\right\} \subset(0,2 / L],\left\{r_{n}\right\} \subset(0,2 \alpha]$, and $\left\{\alpha_{n}\right\},\left\{\beta_{n}\right\},\left\{\gamma_{n}\right\},\left\{\delta_{n}\right\}$ are four sequences in $[0,1]$ such that:

(i) $0<\liminf _{n \rightarrow \infty} \lambda_{n} \leq \limsup \sup _{n \rightarrow \infty} \lambda_{n}<2 / L$ and $\lim _{n \rightarrow \infty}\left(\lambda_{n}-\lambda_{n+1}\right)=0$;

(ii) $0<\liminf _{n \rightarrow \infty} r_{n} \leq \limsup _{n \rightarrow \infty} r_{n}<2 \alpha$ and $\lim _{n \rightarrow \infty}\left(r_{n}-r_{n+1}\right)=0$;

(iii) $\beta_{n}+\gamma_{n}+\delta_{n}=1$ and $\left(\gamma_{n}+\delta_{n}\right) k \leq \gamma_{n}<\delta_{n}$ for all $n \geq 0$;

(iv) $\lim _{n \rightarrow \infty} \alpha_{n}=0$ and $\sum_{n=0}^{\infty} \alpha_{n}=\infty$;

(v) $0<\liminf _{n \rightarrow \infty} \beta_{n} \leq \lim \sup _{n \rightarrow \infty} \beta_{n}<1$ and $\liminf _{n \rightarrow \infty} \delta_{n}>0$;

(vi) $\lim _{n \rightarrow \infty}\left(\gamma_{n+1} /\left(1-\beta_{n+1}\right)-\gamma_{n} /\left(1-\beta_{n}\right)\right)=0$.

Then, $\left\{x_{n}\right\},\left\{y_{n}\right\}$ and $\left\{z_{n}\right\}$ converge strongly to the same point $x^{*}=P_{\mathrm{Fix}(S) \cap \Omega \cap V I}(C, A) u$.

Proof. In Theorem 3.2, putting $\Theta=0, \varphi=0$ and $F=A$, the following relation

$$
\Theta\left(z_{n}, y\right)+\varphi(y)-\varphi\left(z_{n}\right)+\left\langle F x_{n}, y-z_{n}\right\rangle+\frac{1}{r_{n}}\left\langle y-z_{n}, z_{n}-x_{n}\right\rangle \geq 0, \quad \forall y \in C,
$$

is reduced to

$$
\left\langle A x_{n}, y-z_{n}\right\rangle+\frac{1}{r_{n}}\left\langle y-z_{n}, z_{n}-x_{n}\right\rangle \geq 0, \quad \forall y \in C
$$

This implies that

$$
\left\langle y-z_{n}, x_{n}-r_{n} A x_{n}-z_{n}\right\rangle \leq 0, \quad \forall y \in C
$$

So, it follows that $z_{n}=P_{C}\left(x_{n}-r_{n} A x_{n}\right)$ for all $n \geq 0$. Then, by Theorem 3.2, we obtain the desired result.

Let $T: C \rightarrow C$ be a $\tilde{k}$-strictly pseudocontractive mapping. For recent convergence result for strictly pseudocontractive mappings, we refer to Zeng et al. [38]. Putting $F=I-T$, we know that

$$
\|(I-F) x-(I-F) y\|^{2} \leq\|x-y\|^{2}+\tilde{k}\|F x-F y\|^{2}, \quad \forall x, y \in C .
$$

Note that

$$
\|(I-F) x-(I-F) y\|^{2}=\|x-y\|^{2}+\|F x-F y\|^{2}-2\langle x-y, F x-F y\rangle .
$$

Hence

$$
\langle x-y, F x-F y\rangle \geq \frac{1-\tilde{k}}{2}\|F x-F y\|^{2}, \quad \forall x, y \in C .
$$

This implies that the mapping $F=I-T$ is $((1-\tilde{k}) / 2)$-inverse-strongly monotone. 
Corollary 3.7. Let $C$ be a nonempty bounded closed convex subset of a real Hilbert space $H$. Let $\Theta$ : $\mathrm{C} \times \mathrm{C} \rightarrow \mathbf{R}$ be a bifunction satisfying conditions (H1)-(H4) and $\varphi: C \rightarrow \mathbf{R}$ a lower semicontinuous and convex function with assumptions (A1) or (A2). Suppose the minimization (1.5) is consistent and let $\Omega$ denote its solution set. Assume the gradient $\nabla f$ is L-Lipschitzian with constant $L>0$ and $T$ : $C \rightarrow C$ is a $\tilde{k}$-strictly pseudocontractive mapping. Let $S: C \rightarrow C$ be a $k$-strictly pseudocontractive mapping such that $\operatorname{Fix}(S) \cap \Omega \cap \mathrm{GMEP} \neq \emptyset$, where $F=I-T$. For fixed $u \in C$ and given $x_{0} \in C$ arbitrarily, let the sequences $\left\{x_{n}\right\},\left\{y_{n}\right\}$, and $\left\{z_{n}\right\}$ be generated iteratively by

$$
\begin{gathered}
z_{n}=T_{r_{n}}^{(\Theta, \varphi)}\left(\left(1-r_{n}\right) x_{n}+r_{n} T x_{n}\right), \\
y_{n}=\alpha_{n} u+\left(1-\alpha_{n}\right) P_{C}\left(z_{n}-\lambda_{n} \nabla f\left(z_{n}\right)\right), \\
x_{n+1}=\beta_{n} x_{n}+\gamma_{n} P_{C}\left(z_{n}-\lambda_{n} \nabla f\left(z_{n}\right)\right)+\delta_{n} S y_{n}, \quad \forall n \geq 0,
\end{gathered}
$$

where $\left\{\lambda_{n}\right\} \subset(0,2 / L],\left\{r_{n}\right\} \subset(0,1-\tilde{k}]$ and $\left\{\alpha_{n}\right\},\left\{\beta_{n}\right\},\left\{\gamma_{n}\right\},\left\{\delta_{n}\right\}$ are four sequences in $[0,1]$ such that

(i) $0<\liminf _{n \rightarrow \infty} \lambda_{n} \leq \lim \sup _{n \rightarrow \infty} \lambda_{n}<2 / L$ and $\lim _{n \rightarrow \infty}\left(\lambda_{n}-\lambda_{n+1}\right)=0$;

(ii) $0<\liminf _{n \rightarrow \infty} r_{n} \leq \limsup _{n \rightarrow \infty} r_{n}<1-\tilde{k}$ and $\lim _{n \rightarrow \infty}\left(r_{n}-r_{n+1}\right)=0$;

(iii) $\beta_{n}+\gamma_{n}+\delta_{n}=1$ and $\left(\gamma_{n}+\delta_{n}\right) k \leq \gamma_{n}<\delta_{n}$ for all $n \geq 0$;

(iv) $\lim _{n \rightarrow \infty} \alpha_{n}=0$ and $\sum_{n=0}^{\infty} \alpha_{n}=\infty$;

(v) $0<\liminf _{n \rightarrow \infty} \beta_{n} \leq \limsup \sup _{n \rightarrow \infty} \beta_{n}<1$ and $\liminf _{n \rightarrow \infty} \delta_{n}>0$;

(vi) $\lim _{n \rightarrow \infty}\left(\gamma_{n+1} /\left(1-\beta_{n+1}\right)-\gamma_{n} /\left(1-\beta_{n}\right)\right)=0$.

Then, $\left\{x_{n}\right\},\left\{y_{n}\right\}$ and $\left\{z_{n}\right\}$ converge strongly to the same point $x^{*}=P_{\operatorname{Fix}(S) \cap \Omega n G M E P} u$.

Proof. Since $T$ is a $\tilde{k}$-strictly pseudocontractive mapping, the mapping $F=I-T$ is $(1-\tilde{k}) / 2$ inverse-strongly monotone. In this case, put $\alpha=(1-\tilde{k}) / 2$. Then, we conclude that

$$
z_{n}=T_{r_{n}}^{(\Theta, \varphi)}\left(x_{n}-r_{n} F x_{n}\right)=T_{r_{n}}^{(\Theta, \varphi)}\left(x_{n}-r_{n}(I-T) x_{n}\right)=T_{r_{n}}^{(\Theta, \varphi)}\left(\left(1-r_{n}\right) x_{n}+r_{n} T x_{n}\right) .
$$

So, by Theorem 3.2, we obtain the desired result.

\section{Acknowledgments}

This research was partially supported by the National Science Foundation of China (11071169), Innovation Program of Shanghai Municipal Education Commission (09ZZ133), Leading Academic Discipline Project of Shanghai Normal University (DZL707), Ph.D. Program Foundation of Ministry of Education of China (20070270004), Science and Technology Commission of Shanghai Municipality Grant (075105118), and Shanghai Leading Academic Discipline Project (S30405). This research was partially supported by a Grant from NSC 1012115-M-037-001.

\section{References}

[1] F. E. Browder and W. V. Petryshyn, "Construction of fixed points of nonlinear mappings in Hilbert space," Journal of Mathematical Analysis and Applications, vol. 20, pp. 197-228, 1967. 
[2] G. Marino and H. K. Xu, "Weak and strong convergence theorems for strict pseudo-contractions in Hilbert spaces," Journal of Mathematical Analysis and Applications, vol. 329, no. 1, pp. 336-346, 2007.

[3] H. K. Xu, "Averaged mappings and the gradient-projection algorithm," Journal of Optimization Theory and Applications, vol. 150, no. 2, pp. 360-378, 2011.

[4] J. W. Peng and J. C. Yao, "A new hybrid-extragradient method for generalized mixed equilibrium problems, fixed point problems and variational inequality problems," Taiwanese Journal of Mathematics, vol. 12, no. 6, pp. 1401-1432, 2008.

[5] Y. Yao, Y. C. Liou, and J. C. Yao, "New relaxed hybrid-extragradient method for fixed point problems, a general system of variational inequality problems and generalized mixed equilibrium problems," Optimization, vol. 60, no. 3, pp. 395-412, 2011.

[6] L. C. Ceng and J. C. Yao, "A relaxed extragradient-like method for a generalized mixed equilibrium problem, a general system of generalized equilibria and a fixed point problem," Nonlinear Analysis, vol. 72, no. 3-4, pp. 1922-1937, 2010.

[7] L. C. Ceng and J. C. Yao, "A hybrid iterative scheme for mixed equilibrium problems and fixed point problems," Journal of Computational and Applied Mathematics, vol. 214, no. 1, pp. 186-201, 2008.

[8] S. Takahashi and W. Takahashi, "Strong convergence theorem for a generalized equilibrium problem and a nonexpansive mapping in a Hilbert space," Nonlinear Analysis, vol. 69, no. 3, pp. 1025-1033, 2008.

[9] L. C. Ceng, S. Al-Homidan, Q. H. Ansari, and J. C. Yao, “An iterative scheme for equilibrium problems and fixed point problems of strict pseudo-contraction mappings," Journal of Computational and Applied Mathematics, vol. 223, no. 2, pp. 967-974, 2009.

[10] J. W. Peng, "Iterative algorithms for mixed equilibrium problems, strict pseudocontractions and monotone mappings," Journal of Optimization Theory and Applications, vol. 144, no. 1, pp. 107-119, 2010.

[11] E. Blum and W. Oettli, "From optimization and variational inequalities to equilibrium problems," The Mathematics Student, vol. 63, no. 1-4, pp. 123-145, 1994.

[12] A. Tada and W. Takahashi, "Weak and strong convergence theorems for a nonexpansive mapping and an equilibrium problem," Journal of Optimization Theory and Applications, vol. 133, no. 3, pp. 359-370, 2007.

[13] L. C. Ceng and J. C. Yao, "Hybrid viscosity approximation schemes for equilibrium problems and fixed point problems of infinitely many nonexpansive mappings," Applied Mathematics and Computation, vol. 198, no. 2, pp. 729-741, 2008.

[14] H. Iiduka and W. Takahashi, "Strong convergence theorem by a hybrid method for nonlinear mappings of nonexpansive and monotone type and applications," Advances in Nonlinear Variational Inequalities, vol. 9, no. 1, pp. 1-10, 2006.

[15] L. C. Ceng, S. Schaible, and J. C. Yao, "Implicit iteration scheme with perturbed mapping for equilibrium problems and fixed point problems of finitely many nonexpansive mappings," Journal of Optimization Theory and Applications, vol. 139, no. 2, pp. 403-418, 2008.

[16] G. Bigi, M. Castellani, and G. Kassay, "A dual view of equilibrium problems," Journal of Mathematical Analysis and Applications, vol. 342, no. 1, pp. 17-26, 2008.

[17] G. M. Korpelevič, "An extragradient method for finding saddle points and for other problems," Èkonomika i Matematicheskie Metody, vol. 12, no. 4, pp. 747-756, 1976.

[18] L. C. Zeng and J. C. Yao, "Strong convergence theorem by an extragradient method for fixed point problems and variational inequality problems," Taiwanese Journal of Mathematics, vol. 10, no. 5, pp. 1293-1303, 2006.

[19] M. Aslam Noor, "Some developments in general variational inequalities," Applied Mathematics and Computation, vol. 152, no. 1, pp. 199-277, 2004.

[20] Y. Censor, A. N. Iusem, and S. A. Zenios, "An interior point method with Bregman functions for the variational inequality problem with paramonotone operators," Mathematical Programming, vol. 81, no. 3, pp. 373-400, 1998.

[21] Y. Yao, Y. C. Liou, and S. M. Kang, "Approach to common elements of variational inequality problems and fixed point problems via a relaxed extragradient method," Computers $\mathcal{E}$ Mathematics with Applications, vol. 59, no. 11, pp. 3472-3480, 2010.

[22] N. Nadezhkina and W. Takahashi, "Strong convergence theorem by a hybrid method for nonexpansive mappings and Lipschitz-continuous monotone mappings," SIAM Journal on Optimization, vol. 16, no. 4, pp. 1230-1241, 2006. 
[23] W. Takahashi and M. Toyoda, "Weak convergence theorems for nonexpansive mappings and monotone mappings," Journal of Optimization Theory and Applications, vol. 118, no. 2, pp. 417-428, 2003.

[24] I. Yamada, "The hybrid steepest descent method for the variational inequality problem over the intersection of fixed point sets of nonexpansive mappings," in Inherently Parallel Algorithms in Feasibility and Optimization and Their Applications, D. Butnariu, Y. Censor, and S. Reich, Eds., vol. 8, pp. 473-504, Kluwer Academic, Dordrecht, The Netherlands, 2001.

[25] N. Nadezhkina and W. Takahashi, "Weak convergence theorem by an extragradient method for nonexpansive mappings and monotone mappings," Journal of Optimization Theory and Applications, vol. 128, no. 1, pp. 191-201, 2006.

[26] L. C. Zeng and J. C. Yao, "Mixed projection methods for systems of variational inequalities," Journal of Global Optimization, vol. 41, no. 3, pp. 465-478, 2008.

[27] L. C. Ceng, S. M. Guu, and J. C. Yao, "A general iterative method with strongly positive operators for general variational inequalities," Computers \& Mathematics with Applications, vol. 59, no. 4, pp. 1441-1452, 2010.

[28] P. L. Combettes and S. A. Hirstoaga, "Equilibrium programming in Hilbert spaces," Journal of Nonlinear and Convex Analysis, vol. 6, no. 1, pp. 117-136, 2005.

[29] L. C. Zeng, N. C. Wong, and J. C. Yao, "Convergence analysis of modified hybrid steepest-descent methods with variable parameters for variational inequalities," Journal of Optimization Theory and Applications, vol. 132, no. 1, pp. 51-69, 2007.

[30] T. Suzuki, "Strong convergence of Krasnoselskii and Mann's type sequences for one-parameter nonexpansive semigroups without Bochner integrals," Journal of Mathematical Analysis and Applications, vol. 305, no. 1, pp. 227-239, 2005.

[31] J. B. Baillon and G. Haddad, "Quelques propriétés des opérateurs angle-bornés et $n$-cycliquement monotones," Israel Journal of Mathematics, vol. 26, no. 2, pp. 137-150, 1977.

[32] C. Byrne, "A unified treatment of some iterative algorithms in signal processing and image reconstruction," Inverse Problems, vol. 20, no. 1, pp. 103-120, 2004.

[33] S. Reich, "Weak convergence theorems for nonexpansive mappings in Banach spaces," Journal of Mathematical Analysis and Applications, vol. 67, no. 2, pp. 274-276, 1979.

[34] H. K. Xu and T. H. Kim, "Convergence of hybrid steepest-descent methods for variational inequalities," Journal of Optimization Theory and Applications, vol. 119, no. 1, pp. 185-201, 2003.

[35] L. C. Ceng, H. K. Xu, and J. C. Yao, "The viscosity approximation method for asymptotically nonexpansive mappings in Banach spaces," Nonlinear Analysis, vol. 69, no. 4, pp. 1402-1412, 2008.

[36] K. Goebel and W. A. Kirk, Topics in Metric Fixed Point Theory, vol. 28 of Cambridge Studies in Advanced Mathematics, Cambridge University Press, Cambridge, UK, 1990.

[37] R. T. Rockafellar, "On the maximality of sums of nonlinear monotone operators," Transactions of the American Mathematical Society, vol. 149, pp. 75-88, 1970.

[38] L. C. Zeng, N. C. Wong, and J. C. Yao, "Strong convergence theorems for strictly pseudocontractive mappings of Browder-Petryshyn type," Taiwanese Journal of Mathematics, vol. 10, no. 4, pp. 837-849, 2006. 


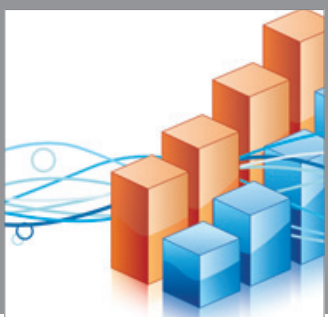

Advances in

Operations Research

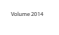

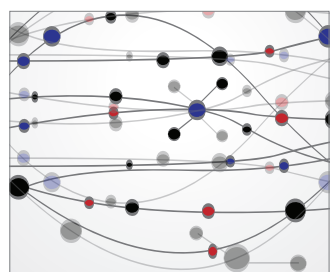

\section{The Scientific} World Journal
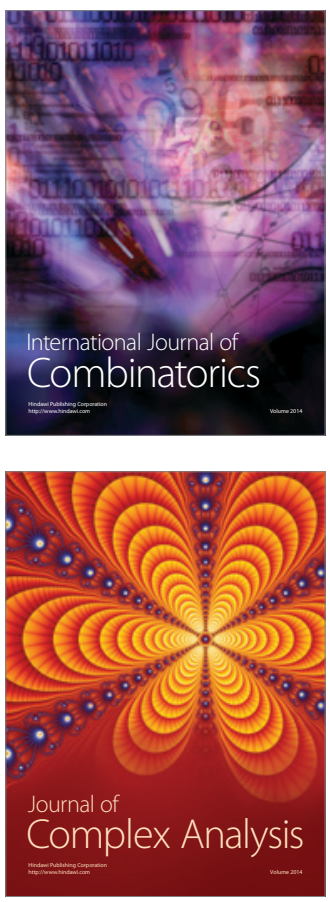

International Journal of

Mathematics and

Mathematical

Sciences
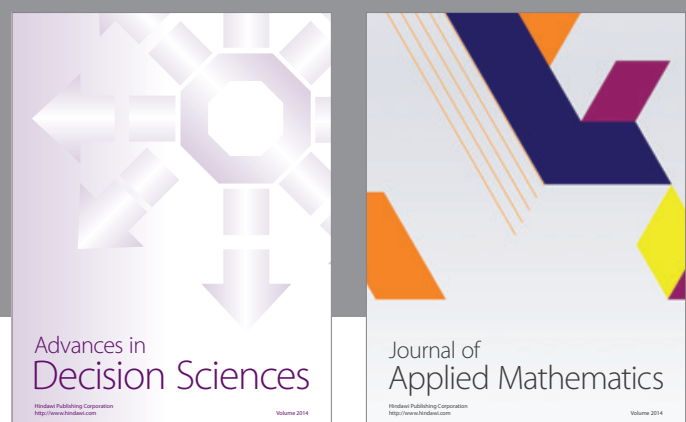

Journal of

Applied Mathematics
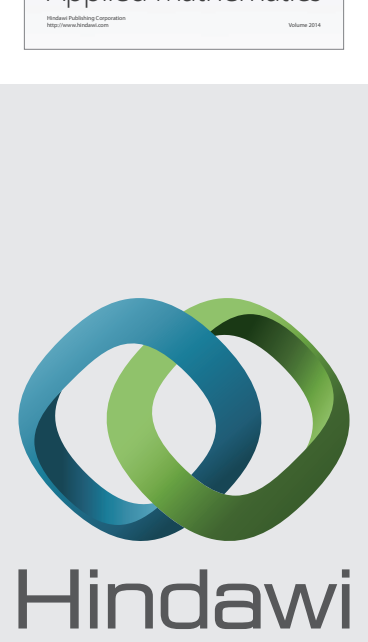

Submit your manuscripts at http://www.hindawi.com
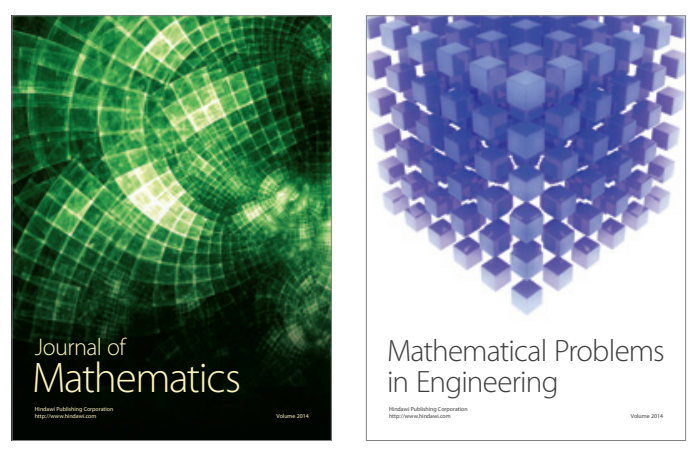

Mathematical Problems in Engineering
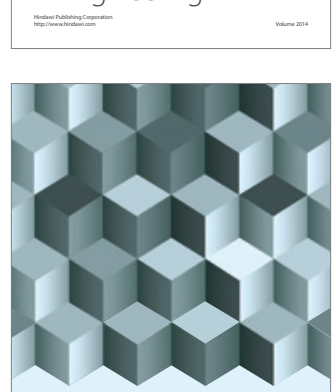

Journal of

Function Spaces
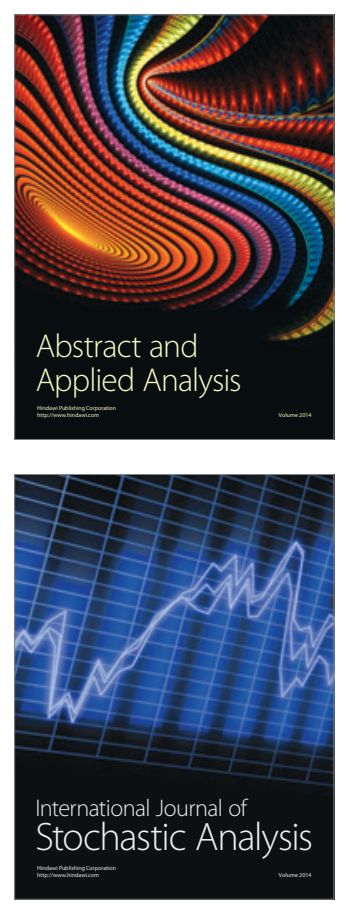

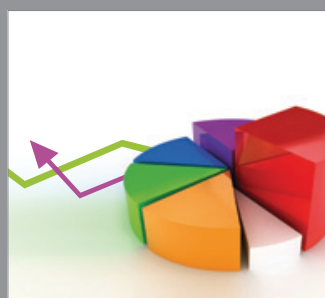

ournal of

Probability and Statistics

Promensencen
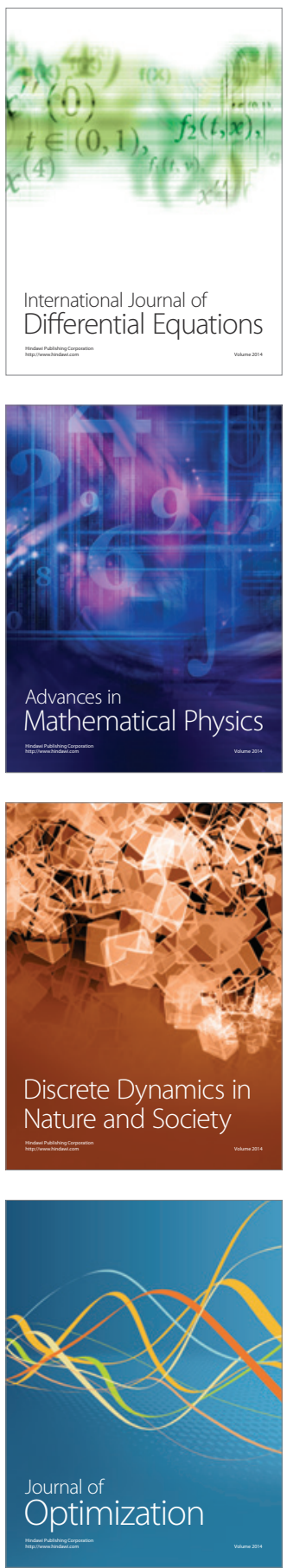